\title{
Die Nesselkapseln der Cnidaria, mit besonderer Berücksichtigung der Hydroida
}

\author{
I. Klassifikation und Bedeutung für die Systematik und Evolution ${ }^{1}$
}

\author{
BERNHARD WERNER \\ Biologische Anstalt Helgoland, Zentrale, Hamburg-Altona
}

\begin{abstract}
The nematocysts of the Cnidaria, with special reference to the Hydroida. I. Classification and importance for systematics and evolution. Long-term investigations on the ecology, morphology and systematics of hydroids, both of the North Sea and the Mediterranean, confirmed the results of earlier authors that most species differ in number and quality of their nematocysts. The classification by WeILL (1934) facilitates a satisfactory diagnosis of the different types of nematocysts present in marine Cnidarians; it is based on morphological characteristics of the discharged nematocysts and comprises 20 types of main- and subcategories, two of which are newly introduced and defined in the present paper. On the basis of earlier results by other authors and our own observations, the qualitative and quantitative distribution of the various types of nematocysts in different systematic units is listed. On this basis, an evaluation is made of the general importance of the nematocysts for taxonomy and evolution of the Cnidaria and their sub-units. The differences in qualitative and quantitative distribution and in the degree of differentiation of the various nematocyst types do not represent characteristics of absolute validity. However, the presence of special types of nematocysts is a positive criterion, which, in addition to other characters of morphology as well as of development, may be used to establish a natural system. If, on the grounds of usual morphological characteristics, the systematic status of a species remains uncertain, the specificity of its nematocysts may facilitate a decision; examples are presented to illustrate this point. There exists a positive correlation between the qualitative and quantitative diversity of the nematocyst equipment of a given systematic group on the one side and the diversity of the morphological, functional and developmental structures of this group on the other side. Thus the Hydrozoa - the group with the most pronounced differentiation and radiation in morphology and complexity of life histories - possess the greatest number of different types of nematocysts.
\end{abstract}

\section{EINLEITUNG}

Wie seit langem bekannt, sind die Nesselzellen, die kennzeichnenden Bauelemente der Cnidaria, in morphologisch und funktionell verschiedenen Typen ausgebildet. Das gilt insbesondere für die in den Nesselzellen liegenden und von ihnen erzeugten Nesselkapseln; sie stellen das für Beutefang, Abwehr und Anheftung wirksame Zellprodukt dar. Neuere Arbeiten allgemeiner und spezieller Zielsetzung (STEPHenson 1929, WeILL

${ }^{1}$ Herrn Professor Dr. W. v. Buddenbrock $\dagger$ zum 80. Geburtstag gewidmet. 
1934, Papenfuss 1936, Tischbierek 1936, Russell 1938, 1939, 1940, 1953, Carlgren 1940, 1945, Hand 1954a, b, 1955a, b, 1961 und andere; vgl. Pax 1940, Rees 1957b) haben gezeigt, daß Vorhandensein und Verteilung der Kapseltypen von diagnostischer Bedeutung für die Systematik der verschiedenen Gruppen sein können. Das allgemeine Ergebnis, daß die Nesselkapseln eine deutliche Differenzierung von einfacher zu komplizierter gebauten Typen erkennen lassen, wurde außerdem als Stütze für phylogenetische Ableitungen verwendet (HADŽI 1958, 1963).

Im Vergleich zu dem großen Formenreichtum der Cnidaria sind jedoch die Nesselkapseln erst bei relativ wenigen Arten genau bekannt. Daher wurde bei den eigenen Untersuchungen an Hydrozoen der Nordsee und des Mittelmeeres damit begonnen, jede gefundene Art auf ihre Ausstattung mit Nesselkapseln zu prüfen. Wie sich sofort herausstellte, war die in der deutschen Literatur immer noch verwendete Einteilung in Volventen, Glutinanten und Penetranten angesichts der Vielzahl der bei marinen Formen vorkommenden Kapseltypen in keiner Weise ausreichend. Als modernste und beste Klassifikation bot sich die von Weill (1934) an.

Bei langjährigen eingehenden Untersuchungen an mehr als 100 Arten aus allen Gruppen der Cnidaria fand Wenl, daß äußere Merkmale wie Form und Größe der unentladenen Kapsel wegen ihrer Variabilität beim gleichen Typ für eine exakte Identifizierung nicht ausreichen. Ebenso sind die Strukturelemente des Inhaltes der unentladenen Kapsel bei vielen Typen nur schwer erkennbar und gestatten daher keine schnelle und einwandfreie Diagnose. Dagegen bot der Nesselapparat der Nesselkapseln im entladenen $\mathrm{Zustand}$ genügend sichere Unterscheidungsmerkmale, so daß WeiLl auf ihrer genauen Untersuchung und sinnvollen Beschreibung eine logische Kategorisierung aufbauen konnte. Diese Klassifikation war ihm die sichere Grundlage für eine umfassende monographische Bearbeitung der Nesselkapseln hinsichtlich Entwicklung, Morphologie, systematischer und evolutionistischer Bedeutung, wobei die gesamte umfangreiche Literatur über die Erforschung der Nesselzellen und ihrer Funktionen ausführlich berücksichtigt wurde.

Der Wert und die Brauchbarkeit des WeIlLschen Systems beruhen darauf, daß es tatsächlich alle bisher bekannten Typen einwandfrei zu identifizieren und einzuordnen gestattet. Es dürfte auch keinerlei Schwierigkeiten machen, gegebenenfalls neue, noch nicht beschriebene Nesselkapseln in diesem System unterzubringen. Zur Kennzeichnung der Situation sei aber bemerkt, daß zu den von WeILL (1934) aufgestellten Hauptund Unterkategorien mit insgesamt 17 verschiedenen Typen von Nesselkapseln nach allen neueren Arbeiten keine grundsätzlich neuen Haupteinheiten hinzugekommen sind, was am überzeugendsten für das System von WEILL spricht. Dieser Sachverhalt schließt nicht aus, daß nicht noch weitere, bisher unbekannte Untereinheiten (vermutlich aber nur in sehr geringer Zahl) existieren und aufgefunden werden. Das triff beispielsweise für eine Kategorie zu, die von WErLL (1934) bereits nach der Literatur aufgeführt, aber damals von ihm selbst noch nicht durch eigene Beobachtungen bestätigt war. Die Existenz des betreffenden Typs ist erst in jüngster Zeir (WEILL 1964) verifiziert worden (siehe p. 8, Tab. 1; Abb. 20, den Typ der Birhopaloiden). Von anderen Autoren haben nur CARLGREN $(1940,1945)$ und Cutress (1955) für eine bestimmte Kategorie zur besseren Unterscheidung einige Untereinheiten definiert (siehe p. 12). Auch bei den eigenen Untersuchungen, bei denen zahlreiche Hydroiden-Arten 
auf ihre Nesselzellausstattung geprüft wurden, konnte lediglich eine Untereinheit neu entdeckt und beschrieben werden. Außerdem wurde ein weiterer spezieller Typ nach Angaben anderer Autoren, die durch die eigenen Beobachtungen bestätigt wurden, definiert und in das System eingefügt (siehe p. 8, Tab. 1, Anmerkung 3,4). In Ubereinstimmung mit allen neueren Autoren, die sich mit der Unterscheidung von Nesselkapseln beschäftigt haben, kann die Brauchbarkeit des Systems von Werll vollauf bestätigt werden. Daher erscheint die Auffassung gerechtfertigt, daß sich in Zukunft jeder Versuch erübrigt, ein anderes System aufzubauen oder zu benutzen.

Wenn vor allem in der neueren englischsprachigen Literatur alle Autoren bei der Jdentifizierung von Nesselkapseln dem System von WEILL gefolgt sind, so wird das dadurch erleichtert, daß WEILL bei der Namengebung der Kapseltypen von der griechischen Wortbedeutung der verwendeten Merkmale ausgegangen ist. Eine Übertragung in andere Sprachen ist daher ohne Schwierigkeiten möglich, wofür als Beispiel die Übernahme des Systems durch Russelt (1953, dort p. 14 ff., Fig. 8) eben ins Englische dienen kann.

An einer Einführung in die $₫$ altschsprachige Literatur fehlt es bisher. Das hängt vermutlich auch damit zusammen, : aß die grundlegenden Arbeiten von WEILL schwer zugänglich und dadurch nicht allge iein bekannt geworden sind. Bis auf wenige Ausnahmen finden sich auch in den neveren Arbeiten, in Lehr- und Bestimmungsbüchern immer noch die schon erwähnten ältren Bezeichnungen Volventen, Glutinanten, Penetranten. Diese Einteilung, die sich primär auf die Funktion bezieht, mochte ausreichen, solange die Nesselzellen einer einzigen Form, nämlich des Süßwasserpolypen $H y d r a$ berïcksichtigt wurden, für den die Begriffe ja tatsächlich durch P. Schulze (1914, 1922) entwickelt wurden. Unter do: ieweils gleichen Funktionstyp können aber morphologisch ganz verschiedene Formtypen fallen, wie sich sofort zeigte, als man sich nicht auf Hydra beschränkte, sondern auch andere Hydrozoa sowie Cnidaria aus den Klassen Scyphozoa und Anthozoa berücksichtigte, die ja ihre Hauptentfaltung im Meer erfahren haben oder auf das Meer beschränkt sind. Daß der Formenmannigfaltigkeit der marinen Cnidaria eine Vielfalt ihrer Nesselkapseltypen entspricht, und daß für die morphologisch verschiedenen Kapseln nicht die Funktion als Einteilungsprinzip dienen kann, wurde dann auch der Ausgangspunkt für die gründlichen Untersuchungen von WEILL, die in seinem System gipfeln.

Die alten Bezeichnungen haben daher für eine Klassifizierung, deren Aufgabe die Identifizierung der verschiedenen Typen nach eindeutig unterscheidbaren morphologischen Merkmalen sein muß, kaum mehr als historischen Wert. Daher ist es das nächstliegende Ziel der vorliegenden Arbeit, das System von WeILL in die deutschsprachige Literatur einzuführen und auch dem Nichtspezialisten zugänglich zu machen. Eine einwandfreie Terminologie erscheint dafür eine notwendige Voraussetzung. Da die eigenen Untersuchungen vorwiegend an Hydroiden durchgeführt wurden, sollen in erster Linie die Nesselkapseln dieser Gruppe berücksichtigt werden. Indes hätte bei einer allgemeinen Darstellung des WeIllschen Systems eine Beschränkung auf die bei den Hydrozoa beziehungsweise Hydroida vorkommenden Typen mit Redht als Mangel empfunden werden können. Es erschien daher zweckmäßig, die gesamte Mannigfaltigkeit der Nesselkapseln zu berücksichtigen und alle bisher bekannten Typen tabellarisch und bildlich zusammenzustellen. Für die beiden Klassen der Scyphozoa und Anthozoa, aus 
denen von mir selbst bisher nur wenige Formen auf ihre Nesselzellausstattung geprüft werden konnten, schließe ich mich den Resultaten von Wenll 1934, Papenfuss 1936, Tischbierex 1936, Carlgren 1940, 1945 und Hand 1954b, 1955a, b an. Der Mitteilung der genaueren Angaben über die bei den untersuchten Einzelarten vorgefundenen Nesselkapseln sollen dann spätere Arbeiten dienen.

Die Hauptaufgabe ist dabei vorerst, nach dem Vorgehen von WeILL, Russell und anderen durch die genaue Untersuchung möglichst vieler bekannter Arten die zahlreichen vorhandenen Lücken auszufüllen. Aus diesem Grunde ist es auch notwendig, bei jeder Neubeschreibung einer Art in die Diagnose genaue Angaben über die Nesselzellausstattung aufzunehmen. Nur so kann es gelingen, die bisher bekannten Tatsachen auf eine breitere Basis zu stellen und die allgemeineren Regeln aufzufinden, die sich aus dem Besitz und der Verteilung der verschiedenen Typen für die Systematik ergeben. Auf diese Weise wird es dann auch möglich sein, zur Beantwortung der Frage beizutragen, welcher Wert den Nesselkapseln für die Kenntnis und Auffindung phylogenetischer Entwicklungslinien innerhalb des Stammes Cnidaria und seiner Untereinheiten beizumessen ist. WeILl hat zu diesen Fragen bereits in sehr ausführlicher Form Stellung genommen und auf ihre allgemeine Bedeutung hingewiesen. Im Anschluß an die Darstellung des Systems der Nesselkapseln soll daher versucht werden, das bislang vorliegende Tatsachenmaterial auf seinen Aussagewert für die Systematik und Evolution zu prüfen.

Die Nesselkapsel kann als eine der kompliziertesten Zelldifferenzierungen des ganzen Tierreichs gelten (vgl. KüHN 1914, p. 431). Gleichwohl ist trotz zahlreicher älterer und neuerer Arbeiten der Vorgang der Entladung in seinen demisch-physikalischen und physiologischen Grundlagen nach wie vor ungeklärt (vgl. dazu die zusammenfassenden Ubersichten von ReIsinger 1961 und MERgNer 1964). Weitere neue Untersuchungen vor allem von amerikanischer und französischer Seite haben sich besonders um die Aufklärung der chemischen Zusammensetzung des Nesselgiftes bemüht und sich mit dem Feinbau der Nesselzellen und ihrer Strukturelemente unter Anwendung der Elektronenmikroskopie befaßt (vgl. dazu besonders Borsseau 1952, Chapman 1961, Chapman \& Thlney 1959, Lenhofr \& Loomis 1961).

\section{TERMINOLOGIE}

Für die Unterscheidung und Abgrenzung der verschiedenen Kapseltypen auf Grund ihrer morphologischen Strukturen bedarf es einer genauen Terminologie, die eine einwandfreie und unmißverständliche Beschreibung ermöglicht und eine unterschiedliche Kennzeichnung gleicher Strukturen durch verschiedene Autoren ausschließst. Die folgende Terminologie, bei der sich eine kurze Rekapitulation der wichtigsten morphologischen Tatsachen nicht vermeiden läßt, dürfte diesen Erfordernissen gerecht werden.

1. Nesselzelle, Nematoblast, Cnidoblast, Nematocyte, Cnidocyte: Zelle, in der sich die Nesselkapsel entwickelt. Die Nesselzelle besteht aus Plasma mit Kern, Nesselkapsel und Zusatzstrukturen. Zu den Zusatzstrukturen ist einmal der Nesselzellstiel zu 
rechnen, der in vielen - nicht in allen - Fällen vorhanden ist und dann meist eine lange schlanke Form hat; weiterhin das Cnidocil am äußeren Pol und fibrilläre Strukturen innerhalb der Zelle, die der Reizaufnahme und -leitung dienen. Den Fibrillen im Plasma, die sich im Stiel zu einem Bündel vereinigen, wird überdies eine Stützfunktion zugeschrieben. Eine besondere spiralige Struktur im Plasma wird als Lasso (Fadenknäuel) bezeichnet.

In der Literatur werden die Bezeichnungen Nematoblast, Cnidoblast einerseits und Nematocyte, Cnidocyte andererseits meist gleichartig verwendet. Genaugenommen kennzeichnen die Begriffe Cnido- und Nematoblast die Zelle mit der in Bildung begriffenen Nesselkapsel, wohingegen unter Cnido- und Nematocyte die Zelle mit der vollentwickelten Nesselkapsel zu verstehen ist (vgl. WeILL 1934, p. 104). Für unsere $Z_{w}$ ecke ist die Unterscheidung bedeutungslos; für die Klassifizierung ist stets nur die Zelle mit der fertig entwickelten Nesselkapsel von Interesse.

2. Nesselkapsel, Nematocyste, Cnide: wirksames Organell der Nesselzelle.

a) Die unentladene Kapsel. Sie besteht aus der mehrwandigen Kapselhülle mit dem Operculum, dem Deckel, soweit ein solcher vorhanden ist ${ }^{1}$, und dem Kapselinhalt. Elemente des Kapselinhaltes: Kapselflüssigkeit, die meist nicht erkennbar ist, und eingestülpter Nesselapparat. Dieser ist in die Kapselflïssigkeit eingebettet und enthält in seinem Inneren ebenfalls ein flüssiges Sekret. In manchen Fällen finden sich in der Kapsel besondere Inhaltskörper: Plasmadifferenzierungen, deren Funktion nicht bekannt ist (vgl. WEILL, 1934, p. 33 ff.). Ein Teil des Nesselapparates der unentladenen Kapsel kann als Achsenkörper (englisch: axial rod; französisch: corps axiale) ausgebildet sein, wenn er im entladenen Zustand an der Basis ein besonderes, vom terminalen Nesselschlauch gesondertes Formelement besitzt, nämlich den Schaft (siehe unten). Die Nesselkapsel kommt durch den Vorgang der Entladung zur Funktion, der in einer Ausstülpung des Nesselapparates besteht.

b) Die entladene Kapsel. Sie wird gebildet aus der Kapselhïlle mit dem abgehobenen Operculum, soweit ein solches vorhanden ist, und dem ausgestülpten Nesselapparat.

Nesselapparat ${ }^{2}$ : wirksamer Teil der entladenen Kapsel. Nach dem allgemeinen Sprachgebrauch werden unter Nesselzellen auch solche verstanden, die keine eigentlich nesselnde Funktion haben, deren Wirkung also nicht auf der lähmenden und tötenden Wirkung des in der Kapsel enthaltenen Nesselgiftes beruht, sondern auf dem Festhalten der Beute oder dem Anheften an ein Substrat (vgl. Pax 1940, p. 284). Daher erscheint die gegebene Definition des Nesselapparates unabhängig von seiner tatsächlichen Funktion gerechtfertigt. Der Nesselapparat ist der Teil der Kapsel, der die meisten Differenzierungen aufweist, so daß die Unterscheidung seiner Teilstrukturen

1 Das Operculum ist nich bei allen Kapseltyen als gesonderte und deutlich erkennbare Bildung ausdifferenziert (vgl. WeILI 1934, p. 32 f.).

2 Der Begriff "Nesselapparat" wird in anderem, erweitertem Sinne von PAx (1940, p. 284 f.) zur Kennzeidhung der gesamten Nesselzellausstattung einer Art verwendet, also mit ihrem Cnidom gleichgesetzt. Da Mißverständnisse wohl kaum zu befürchten sind, wird hier an der Verwendung des Begriffes "Nesselapparat“ als eines Teiles der einzelnen Nesselkapsel festgehalten. 
die Grundlage für die Klassifikation von WEILL darstellt, wie bereits erwähnt wurde. Der Nesselapparat kann ein Schlauch sein, er kann die Form eines Stabes oder einer Keule haben und kann eine Kombination dieser Formelemente darstellen.

Im einfachsten Fall besteht der Nesselapparat aus einem feinen, kürzeren oder längeren Schlauch, der keinerlei morphologische Strukturelemente oder Differenzierungen aufweist. Bei der überwiegenden Mehrzahl aber besitzt der Nesselapparat besondere Formelemente; vor allem ist ein erweiterter basaler Schaft, früher auch Halsstück genannt (englisch: shaft, butt; französisch: hampe), von dem dünnen Endabschnitt, dem Nesselschlauch, abgesetzt (englisch: tube; französisch: tube nématocystique). Der Nesselschlauch wird häufig auch Faden genannt. Daß es sich dabei nicht um ein solides, sondern um ein hohles Formelement handelt, braucht nicht eigens betont zu werden. Weiterhin ist der Nesselapparat bei der Mehrzahl der Kapseln nicht glatt, sondern ganz oder teilweise mit Dornen besetzt, die an ihm in mehreren, meist 3 rechtsgewundenen Spiralen inserieren. Rechtsgewunden bedeutet im Uhrzeigersinn, wenn man auf die entladene Kapsel parallel zu ihrer Längsachse in der Richtung von der Basis zur Spitze des Schlauches sieht. Die Dornen (englisch: spines; französisch: épines) sind oftmals sehr fein und zart, so daß man besser von Haaren sprechen würde, wie es Werll mit den Bezeichnungen „atriche, basitriche Haplonemen“ auch tatsächlich tut (siehe unten). Nur ein Nesselkapseltyp, der der Stenotelen (früher Penetranten genannt), besitzt am Schaft drei besonders differenzierte und stärker ausgebildete Dornen, die Stiletts.

\section{Die Nesselzellausstattung, das Cnidom.}

Für diesen von Werll geschaffenen Begriff, der in der neueren Literatur häufig Verwendung findet, gibt der Autor folgende Definition: „Le cnidome est défini l'ensemble des nématocystes que possède un Cnidaire à un moment donné de son existence" (1934, p. 650, vgl. p. 351). Damit ist das Cnidom charakterisiert als "Gesamtheit aller Nesselkapseln, die ein Cnidarier in einem bestimmten Augenblick seines Lebens besitzt" ${ }^{"}$. Diese Definition erscheint allerdings nicht ganz eindeutig, da sie offenbar das individuelle Moment nicht ausschließt und sich primär auf die Gesamtzahl aller bei einem Einzeltier überhaupt vorhandenen Nesselkapseln der verschiedenen Kategorien bezieht. Wenn man bedenkt, daß die Nesselzellausstattung einer Meduse von der des Polypen der gleichen Art verschieden sein kann, weil die eine Generation einen bestimmten Kapseltyp besitzt, der der anderen fehlt, so wird klar, daß die Definition in dem Sinne präzisiert werden muß, daß das Cnidom die Gesamtheit allerverschiedenen Nesselkapseltypendarstellt, über die e i n e A r t verf üg t. Entsprechend besitzt eine Species ein Monocnidom, wenn bei ihr nur ein einziger Typ von Nesselkapseln vorkommt. Andere Arten haben ein Bi-, Tri- oder ein Tetracnidom, wenn 2,3 oder 4 verschiedene Typen von Nesselkapseln bei ihnen gefunden werden. In genau diesem Sinne wenden WerLl (1934) und nach ihm die späteren Autoren den Begriff des Cnidom durchweg an, der damit die Nesselzellausstattung einer Art eindeutig kennzeichnet und nur auf dem Speciesniveau sinnvoll ist.

Das Cnidom einer Art darf als vollständig bekannt nur dann gelten, wenn alle Alters- und Entwicklungsstadien, wenn insbesondere bei metagenetischen Formen beide 
Generationen und wenn im Fall des Polymorphismus alle Formelemente des Stockes oder einer Kolonie auf Vorhandensein und Art der Nesselkapseln untersucht sind, worauf WEILL mehrfach hingewiesen hat (1934, pp. 352, 641). Wie wichtig diese Feststellung ist, geht daraus hervor, daß ein spezieller Kapseltyp nur bei einem bestimmten Entwicklungsstadium auftreten kann, so daß er im ganzen Lebenszyklus und in der Generationenfolge weder vorher noch nachher nachweisbar ist.

\section{DIE KLASSIFIKATION DER NESSELKAPSELN NACH WEILL (1934)}

Wie in der Einleitung erwähnt, ist der ausgestülpte Nesselapparat der entladenen Kapsel das morphologische Grundelement, dessen unterscheidbare Teilstrukturen WEILL für den Aufbau seines Systems der Nesselkapseln gedient haben. Uber die älteren Einteilungsversuche hat derselbe Autor ausführlich berichtet, so daß wir uns hier auf die von ihm begründete Klassifikation beschränken können, die in Tabelle 1 wiedergegeben ist.

Bei den Cnidaria lassen sich danach insgesamt 20 verschiedene Arten von Nesselkapseln unterscheiden, die sich auf 2 Haupt- und eine Anzahl von Untereinheiten verteilen. Von WeILL werden die verschiedenen Nesselkapselarten Kategorien genannt. Der Einfachheit halber werden sie im folgenden meist als Nesselkapseltypen (NKTypen) bezeichnet. Die in der Praxis gebräuchliche Benennung, aus der die Identität eines Kapseltyps mit ausreichender Genauigkeit hervorgeht, ist in Tabelle 1 durch Fettdruck hervorgehoben. Daraus ist ersichtlich, daß in den meisten Fällen eine höchstens binominäre Nomenklatur genügt und bei dem Rest eine maximal trinominäre Nomenklatur notwendig ist. Das haben schon Russell (1953) und HaNo (1961) betont; letzterer hat vergleichsweise darauf hingewiesen, daß zur Kennzeichnung einer Spezies ja auch nicht jedesmal über das Genusniveau hinweg alle höheren systematischen Einheiten angegeben werden. Aber auch bei den Eurytelen (14) bis (18) der Tabelle 1 genügt in den meisten Fällen die Angabe, ob sie mikro- oder makrobasischer Natur sind, da die feinere Unterscheidung nach der Art der Bedornung oftmals schwierig ist, zumal wenn es sich um Kapseln geringer Dimensionen handelt. Mit den Definitionen der Tabelle 1 und den Abbildungen 1 bis 20 erscheinen alle Typen ausreichend gekennzeichnet, so daß sich eine ausführliche Beschreibung erübrigt. Nur auf einige allgemeinere Punkte muß zum besseren Verständnis noch näher eingegangen werden.

1. Vorauszuschicken ist, daß die Unterscheidung der beiden Hauptkategorien Astomocniden und Stomocniden nicht ohne weiteres möglich ist, weil bei den Stomocniden die terminale Offnung des meist langen und am Ende feinen Fadens mit den vorhandenen optischen Hilfsmitteln einschließlich des Phasenkontrastverfahrens nicht erkennbar ist. Der Versuch, nach diesem Unterscheidungsmerkmal im Einzelfall $\mathrm{zu}$ einer einwandfreien Diagnose zu gelangen, würde die Anwendung spezieller Färbemethoden, etwa der Vitalfärbung der Nesselkapseln vor der Entladung erfordern (vgl. WeILL 1934, p. 39). Das Vorhandensein beziehungsweise Fehlen der für die Definition wesentlichen Offnung ist daher bei den meisten Nesselzellen beider Kategorien in der Praxis nur indirekt zu erschließen; doch ist diese Schwierigkeit erfahrungsgemäß belanglos, weil die drei Typen der Astomocniden wegen ihrer morphologischen Beson- 
Tabelle 1

Das System der Nesselkapseln nach WeaLL $(1934)^{1}$

A. Astomocniden:

I. Rhopalonemen:

1. Anacrophoren:

2. Acrophoren:

II. Desmonemen:

B. Stomocniden:

I. Haplonemen:

a) atriche:

b) basitriche:

c) merotriche";

d) holotriche:

2. Anisorhizen:

a) homotriche:

b) heterotriche:

II. Heteronemen:

1. Rhabdoiden:

a) Mastigophoren:

a) mikrobasische:

B) makrobasische:

b) Amastigophoren:

a) mikrobasische:

B) makrobasische:

2. Rhopaloiden:

a) Eurytelen:

a) mikrobasische: $\alpha \alpha)$ homotriche:

$\beta \beta)$ heterotriche:

B) makrobasische:

$\alpha \alpha$ ) telotriche:

BB) merotriche:

$\gamma \gamma)$ holotriche

b) Stenotelen:

\section{Birhopaloiden ${ }^{5}$ :}

1. Isorhizen:

Nesselapparat ohne terminale Offnung

Nesselapparat keulenförmig

ohne apikalen Appendix

mit apikalem Appendix

Nesselapparat spiralig aufgewundener, meist kurzer, dicker Faden ("Volventen")

Nesselapparat mit terminalex Offnung

Nesselapparat einfacher Faden, ohne deutlich abgesetzten basalen Schaft

Faden isodiametrisch

Faden in ganzer Länge ohne Dornen („Glutinanten ")

nur basaler Teil des Fadens mit Dornen

ein der Basis genäherter Zwischenteil des Fadens mit Dornen

Faden in ganzer Länge mit Dornen besetzt ("Glutinanten“)

Faden an der Basis deutlich erweitert

alle Dornen gleichartig

Dornen der Fadenbasis stärker entwidkelt

Nesselapparat mit deutlich abgesetztem basalem

Schaft, oder Schatt ohne Faden

Schaft stabförmig, isodiametrisch

Schaft verjüngt, sich in einen deutlich von ihm abgesetzten Faden

Schaft kurz, $\leqq 3 \times$ Kapsellänge

Schaft lang, $\geqq 4 \times$ Kapsellänge

Schaft ohne Faden

Schaft kurz, $\leqq 3 \times K$ apsellänge

Schat lang, $\geq 4 \times K$ apsellänge

Schaft keulenförmig, anisodiametrisch

Schaft am distalen Ende erweitert

Schaft kurz, $\leq 3 \times K$ apsellänge

Dornen des Schaftes sämtlich gleichartig

Dornen des Schaftes ungleichartig

Schaft lang, $\geqq 4 \times$ Kapsellänge

Endteil des Schaftes mit Dornen besetzt

Zwischenstück des Schaftes vor der Enderweiterung mit Dornen besetzt

Schaft in ganzer Länge mit Dornen besetzt

Schaft an der Basis erweitert, 3 Dornen als Stiletts besonders stark entwickelt ("Penetranten“)

Schaft am proximalen und distalen Teil keulen-

förmig erweitert, also langgestreckt hantelförmig

1 Da bereits RusselL (1953, p. 15) die griechischen Grundbedeutungen der WeILlschen Definitionen angegeben hat, kann hier auf ein ausführliches Glossar verzichtet werden.

2 Mit den in Klammern gesetzten Zahlen wird jedem Typ eine Nummer zugeordnet, unter der er auch in den Abbildungen erscheint. Auf diese Weise kann sowohl in dieser Arbeit wie in späteren Beiträgen auf die hier definierten und abgebildeten Nesselkapseltypen eindeutig Bezug genommen werden.

${ }^{3}$ Dieser Typ wird neu eingeführt und in einem späteren Beitrag beschrieben, ver* gleiche p. 29.

4 Von Weill nicht definiert, von Russell (1939, p. 154 ff., Fig. 45 a-d) beschrieben und abgebildet, allerdings irrtümlich als Mastigophoren bezeichnet. Wie Hand (1954a, p. 54 ff. Fig. 3, 5) gezeigt hat, handelt es sich um Eurytelen.

5 Nach WeILL (1964). 
derheiten als solche sofort erkannt und im gegebenen Fall aus dem Kreis der zu prüfenden Typen ausgeschieden werden können.

2. Russel. (1938, p. 159, 161) hat die Vermutung ausgesprochen, daß es sich bei den Anacrophoren (1) und Acrophoren (2), die WeILl ausschließlich für die Siphonophoren signalisiert hat (siehe p. 19, Tabelle 3), möglicherweise um Entwicklungsstadien anderer Kapseltypen handele, nämlich von Desmonemen und Haplonemen. Diese Vermutung ist aber bislang weder von Russell selbst noch von anderen Autoren bestätigt worden. Daher müßte schon aus diesem Grunde bis zum Beweis des Gegenteils an der Existenz der Ana- und Acrophoren festgehalten werden, die auch schon von älteren Autoren beschrieben und abgebildet sind (vergleiche KüHn 1915, Taf. 31, Fig. 4, 14). Darüber hinaus hatte ich neuerdings als Teilnehmer der "Meteor"-Expedition 1964/65 in den Indischen Ozean Gelegenheit, lebendfrisches Material mehrerer Siphonophoren-Arten auf die vorhandenen Nesselkapseln zu prüfen. Dabei konnte der Typ der Anacrophoren (1) im unentladenen und entladenen Zustand beobachtet werden. Form und Struktur stimmten mit den Angaben von WEILL vollständig überein und ließen keinen $Z$ weifel daran aufkommen, daß es sich um einen selbständigen Typ handelt. Das gleiche ist damit auch für die Acrophoren (2) anzunehmen.

3. Wie Tabelle 1 erkennen läßt, entfällt die Hauptmenge der Typen auf die Haplo- und Heteronemen. Der Vollständigkeit halber sei erwähnt, daß die Heteronemen mit Ausnahme der Amastigophoren noch in die beiden größeren Gruppen der Hoplotelen und Anoplotelen aufgegliedert werden können, je nachdem der terminale Schlauch des Nesselapparates bewaffnet, das heißt mit Dornen besetzt ist oder nicht (Wenl 1934, p. 38). Ferner ist zu beachten, daß die Unterscheidung der Typen bei einer Reihe von Haplo- und Heteronemen auch im unentladenen Zustand möglich ist, wenn das Merkmal - Vorhandensein oder Fehlen eines Achsenkörpers - und dessen Ausbildungsgrad für die Diagnose berücksichtigt werden. Nach WeILL $(1934$, p. 49) gilt nämlich die Beziehung:

a) ohne Achsenkörper

b) mit schwach entwickeltem Achsenkörper

c) mit stark entwickeltem Achsenkörper

Haplonemen

Heteronemen

Daher können die Kategorien a) und c) auch im unentladenen Zustand sofort angesprochen werden, nicht aber die Kategorie b). Erfahrungsgemäß lassen darüber hinaus auch zahlreiche andere Typen bei einiger Ubung die Identifizierung im unentladenen Zustand zu. Das gilt ganz besonders für die Desmonemen und Stenotelen, wie auch für die makro- und mikrobasischen Eurytelen.

Andererseits darf nicht unerwähnt bleiben, daß es Fälle gibt, bei denen die Unterscheidung der Typen auch nach dem in seinen morphologischen Merkmalen so eindeutigen Werluschen System Schwierigkeiten bereitet. Das gilt beispielsweise für einige Typen der Haplo- und Heteronemen. So hat schon Russell $(1938,1953)$ bemerkt, daß bei den Thecata die Unterscheidung zwischen den basitrichen Haplonemen (5) und mikrobasischen Mastigophoren (10) auch bei den entladenen Kapseln oft schwierig ist. Die Ursache liegt meist in der sehr geringen Größe der Kapseln dieser Typen. Aus dem gleichen Grunde kann auch die Unterscheidung zwischen den mikrobasischen Eury- 
telen (15) und den mikrobasischen Mastigophoren (10) mit Schwierigkeiten verbunden sein. Doch handelt es sich im ganzen gesehen nur um wenige Ausnahmefälle; sie sprechen keineswegs gegen das System yon WeILl, da sie überdies auch damit zu erklären sind, daß es Ubergänge von einem zum anderen Typ geben kann, worin offenbar evolutionistische Zusammenhänge zum Ausdruck kommen.

4. Die vermutlich gleiche Ursache ist auch für eine weitere Erscheinung anzunehmen. Definitionsgemäß ist der Faden bei den Isorhizen, der ersten Untereinheit der Haplonemen, in ganzer Länge von annähernd der gleichen Dicke. Daß indes diese Eigenschaft nicht bei allen Isorhizen in gleicher Weise voll verwirklicht ist, zeigt der in Abbildung 7 dargestellte Typ der holotrichen Haplonemen (7). Wie die gezeichneten Teilstücke des Fadens erkennen lassen, verjüngt er sich von der Basis bis zur Spitze allmählich, aber doch deutlich. Andererseits ist die basale Erweiterung des Fadens bei den Anisorhizen $(8,9)$ so viel deutlicher ausgeprägt, daß die Trennung in verschiedene Kategorien schon allein nach diesem Merkmal notwendig und gerechtfertigt ist, wozu die Unterschiede in der Bedornung noch hinzukommen.

5. WeILl (1934, p. 48, 50 ff.) hat darauf aufmerksam gemacht, daß auch bei den atrichen Haplonemen (4) die Oberfläche des Fadens nicht völlig glatt, sondern mit feinen Spiralstrukturen („bandelettes hélicoidales“) versehen ist und daß Ubergänge zwischen den atrichen und holotrichen Haplonemen (7) vorkommen. Diese Angaben konnten bei den eigenen Untersuchungen bestätigt werden. Es drängt sich damit die Vermutung auf, daß es "echte" atriche Haplonemen nicht gibt. Diese Vermutung wurde inzwischen von anderer Seite bestätigt. Auf dem Symposion on the Cnidaria and their evolution of The Zoological Society of London, 3. bis 4. März 1965, ergab die Diskussion im Anschluß an den Vortrag von L. E. R. Picken und R. J. Skaer: "A review of nematocyst researches“, daß sich die atrichen Haplonemen bei der elektronenoptischen Untersuchung als mit feinsten Härchen versehen erweisen. Trotz dieses

Abb. 1 bis 11: Die Nesselkapseln der $C$ nidaria. Die Abbildungen geben jeweils die unentladene (a) und die entladene Kapsel (b) wieder. Vergrößerungen der mit dem Abbéschen Zeichenapparat gezeichneten Originale X 1700. Die mehrwandige Kapselhülle ist einfach gezeichnet

1 Anacrophore von Dipbyes spiralis (Siphonophora), nach Wenl. (1934, p. 40, Fig. 22 a, b).

2 Acrophore von Forskalia spec. (Siphonophora), nach Werl (1934, p. 40, Fig. 23 a, c).

3 Desmoneme, schlanke Form, von Cladonema radiatum, Meduse (Hydroida Athecata), Original.

3' Desmoneme, häufigste Form, von Pelmatobydra oligactis (Hydroida Hydrina). Original.

3" Desmoneme, mit langem Faden, von Proboscidactyla, Polyp (syn. Lar) (Hydroida Limnohydrina), kombiniert nach Russeil (1938, p. 155, Fig. 45 j) und Hand (1954a, p. 57, Fig. $5 \mathrm{~h}$ ).

4 atriche Haploneme von Eirene viridula, Polyp (Hydroida Thecata), Original.

5 basitriche Haploneme von Eirene viridula, Polyp (Hydroida Thecata), Original.

6 merotriche Haploneme von Eucheilota maculata, Meduse (Hydroida Thecata), Original. 7 holotriche Haploneme von Pelmatohydra braneri (Hydroida Hydrina), Original.

8 homotriche Anisorhize von einer Siphonophore, nad WEILL (1934, p. 55. Fig. 41 a, b). 9 heterotriche Anisorhize von Euphysa aurata, Meduse (Hydroida Athecata), Original.

10 mikrobasische Mastigophore von Eucheilota maculata, Meduse (Hydroida Thecata), Original.

11 makrobasische Mastigophore von Millepora spec. (Hydroida Athecata), nach WErLL (1934, p. 65, Fig. 55 a, b). 
Nesselkapseln der Cnidaria
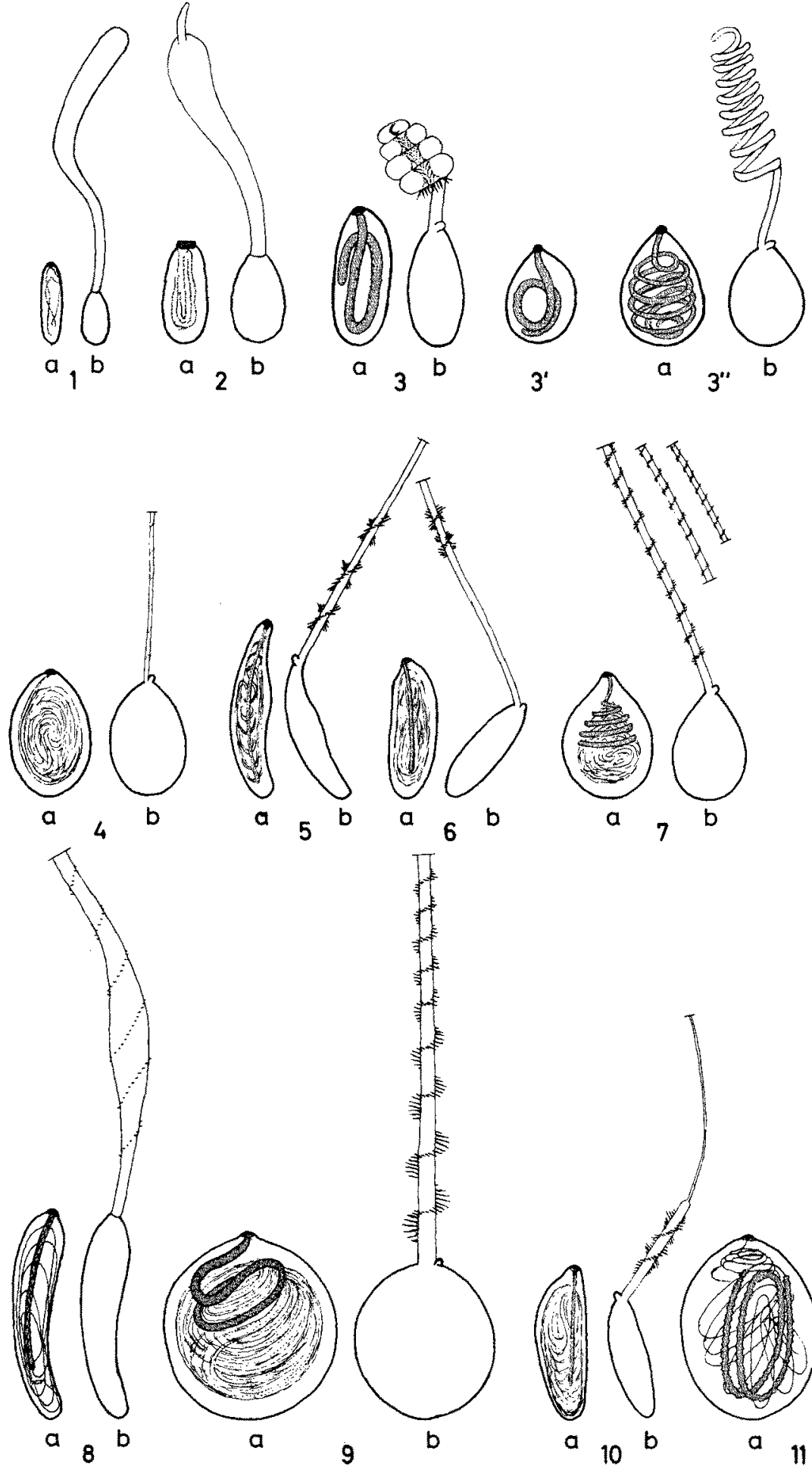
positiven Befundes ist aber der Unterschied in der Oberflächenstruktur zwischen den atrichen Haplonemen einerseits, den basi-, mero- und holotrichen Haplonemen mit ihren deutlich ausgebildeten und leicht erkennbaren Dornen beziehungsweise Haaren andererseits so erheblich, daß der Typ der atrichen Haplonemen auch in Zukunft seine Berechtigung behält.

6. Carlgren $(1940,1945)$ und Cutress (1955) haben den Typ der Mastigophoren (10), (11) in weitere Untereinheiten aufgeteilt (mikrobasische b- und p-Mastigophoren nach CARLGREN, mikrobasische q-Mastigophoren und makrobasische p-Mastigophoren nach Cutress). Diese Untertypen werden von den genannten Autoren aber nur für die Anthozoen beschrieben und sind meines Wissens für Vertreter der Hydrozoen noch nicht signalisiert. Auf eine eingehende Berücksichtigung kann daher verzichtet werden.

7. Cutress (1955) verneint die Existenz der mikrobasischen (12) und makrobasischen (13) Amastigophoren vollständig. Nach seiner Ansicht haben die Amastigophoren sämtlich am Schaft einen kurzen feinen Endfaden, wären also in Wirklichkeit Mastigophoren. Daß die Untersuchungen von CuTress nicht eindeutig genug sind, hat schon HAND (1961, p. 189 ff.) erwähnt, der auch auf Grund elektronenmikroskopischer Befunde zur Zurückweisung der Auffassung von Cutress gelangt. Nach meinen eigenen Beobachtungen an Hydroiden haben die Mastigophoren stets einen langen Faden, so daß die Amastigophoren, selbst wenn die Spitze ihres Nesselapparates in einen kurzen feinen Faden ausläuft, als eigener Typ von den Mastigophoren getrennt werden müssen. WeIL hat brieflich (2. Dezember 1963) überdies hervorgehoben, daß der Nesselapparat der Amastigophoren nach Länge und Bedornung sowohl im unentladenen wie im entladenen Zustand nur mit dem $S \mathrm{ch}$ a f $\mathrm{t}$ der Mastigophoren vergleichbar ist, was ebenfalls für die Sonderung in getrennte Typen spricht.

8. Schließlich muß erwähnt werden, daß die Anthozoa - und nur diese Gruppe über einen weiteren Typ von Kapseln verfügen, die Spirocysten (Abb, 21). Es handelt sich um langgestreckte, zylindrische, sehr dünnwandige Kapseln, die im Inneren durch eine charakteristische Spiralstruktur des Fadens ausgezeichnet und dadurch sofort erkennbar sind. Außerdem unterscheiden sie sich von den anderen Nesselkapsel-

Abb. 12 bis 19: Die Nesselkapseln de: Cnidaria (Fortsetzung)

12 mikrobasische Amastigophore von Sagartia miniata (Hexacorallia Actiniaria), nach WeILL (1934, p. 69, Fig. 59 a, b).

13 makrobasische Amastigophore von Lebrunia danae (Hexacorallia Actiniaria) nach Werli (1934, p. 69, Fig. 62 a, b).

14 homotriche mikrobasische Eurytele von einer Anthomeduse (Hydroida Athecata), nach WeILL (1934, p. 74, Fig. 64 a, b).

15 heterotriche mikrobasische Eurytele von Gonionemus vertens, Polyp (Hydroida Limnohydrina), Original.

16 telotriche makrobasische Eurytele von Pteroclava krempfi, Meduse (Hydroida Athecata), nach WEILL (1934 p. 79, Fig. 72 a, b).

17 merotriche makrobasische Eurytele von Zanclea costata (syn. Gemmaria gemmosa), Meduse (Hydroida Athecata), nach Werll (1934, p. 79, Fig. 73 a, b).

18 holotriche makrobasische Eurytele von Gonionemus vertens, Jungmeduse (Hydroida Limnohydrina), Original.

19 Stenotele von Coryne tubulosa, Meduse (Hydroida Athecata), Original. 
Nesselkapseln der Cnidaria

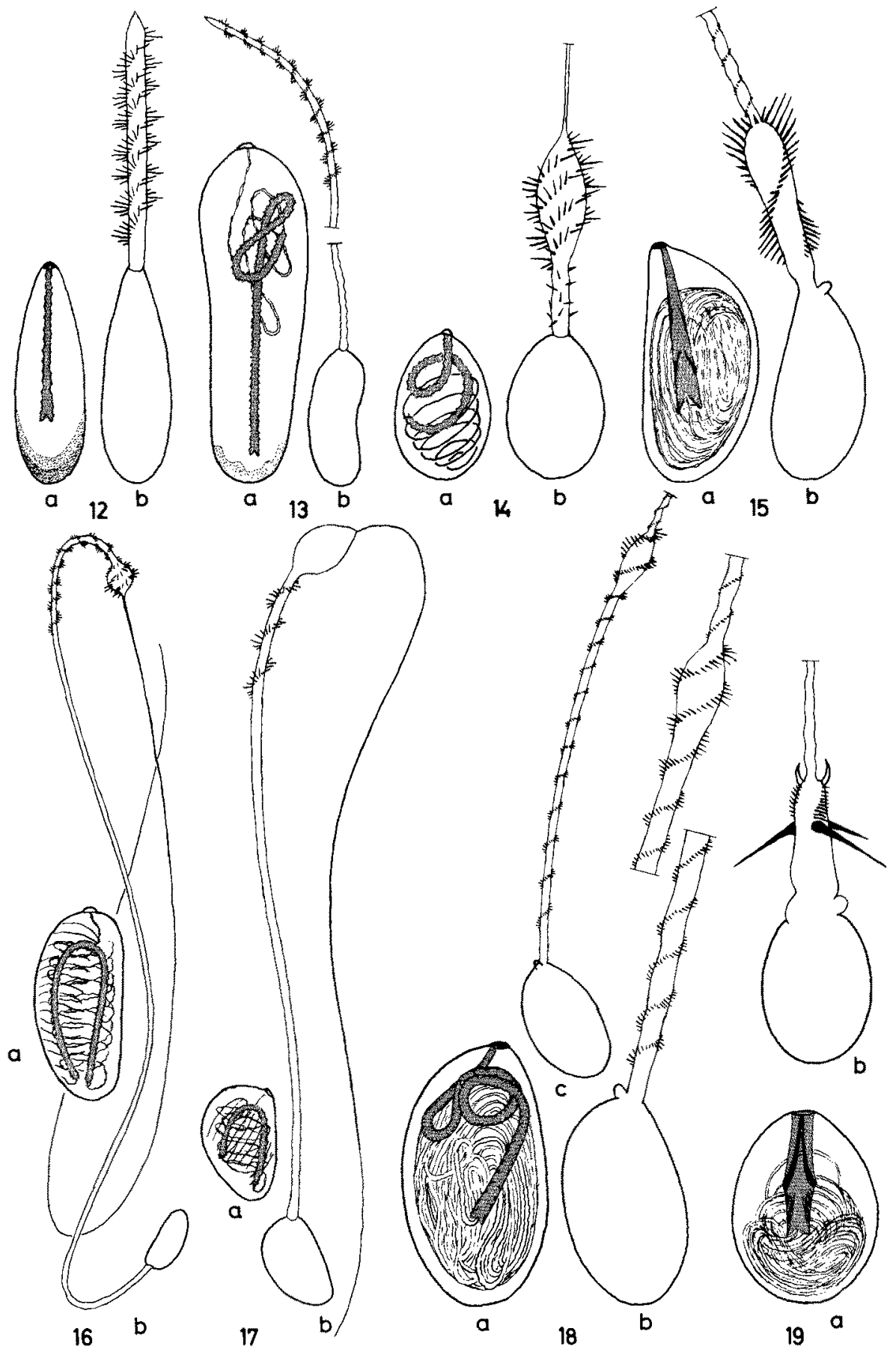


arten durch ein unterschiedliches Verhalten gegenuiber Farbstoffen. Dieser Typ ist von WEILL bewußt aus seiner Klassifikation ausgeschlossen, da nach seinen Angaben die Kenntnisse über Bau und Funktion sowie über die Entwicklung noch immer als unvollständig bezeichnet werden müssen (Weill 1934, p. 325 ff.).

Neuerdings hat Cutress (1955) die Ansicht vertreten, daß es sich bei den Spirocysten nach Bau und Funktion um nichts anderes als einen besonderen Typ von Nesselkapseln handele. Seine Beweisführung erscheint allerdings noch keineswegs ausreichend begründet (vgl. HaND 1961), so daß eine genaue Nachprüfung notwendig ist.
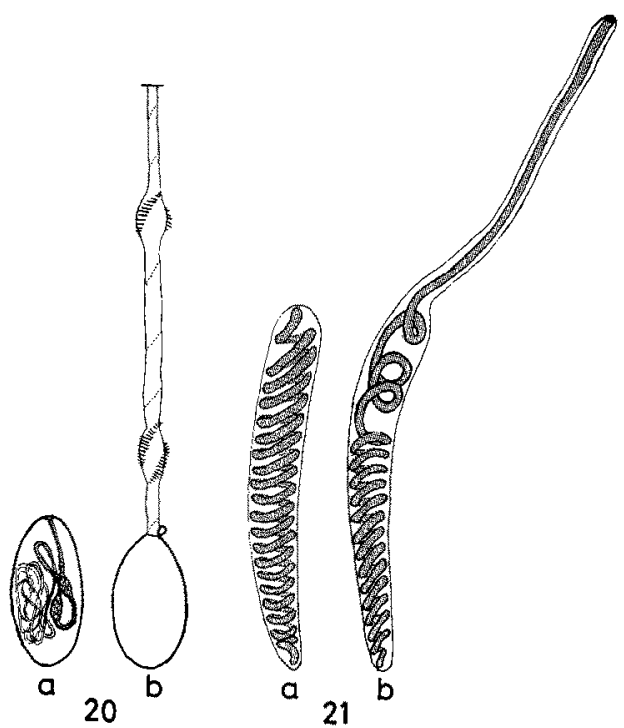

Abb. 20 bis 21: Die Nesselkapseln der Cnidaria (Fortsetzung)

20 Birhopaloide von Apolemia wvaria (Siphonophora), nach KüHn (1915, Taf. 31, Fig. 16, 17). 21 Spirocyste von Peachia hastata (Hexacorallia Actiniaria), Original,

\section{BEI DEN SYSTEMATISCHEN EINHEITEN DER CNIDARIA VORKOMMENDE NESSELKAPSELN UND IHRE BEDEUTUNG FÚR DIE SYSTEMATIK UND EVOLUTION}

Die Arbeiten von Weill, Papenfuss, Tischbierek, Russell, Carlgren, Hand und anderen haben zunächst gezeigt, $\mathrm{da} ß$ die Formen aus den verschiedenen taxonomischen Einheiten der Cnidaria recht erhebliche Unterschiede in ihrer Nesselzellausstattung aufweisen. Das gilt einmal für die Ty p ve r s c hi eden heit der bei den Species vorkommenden Nesselkapseln, wie auch für die $\mathrm{Z}$ a hl verschiedener NK - Ty pen, über die sie verfügen. Wie einleitend schon kurz erwähnt, kann weiterhin als allgemeines Ergebnis gelten, daß die Nesselkapseln für die Systematik von diagnostischem Wert sind. Es soll versucht werden, den Geltungsbereich dieser Aussage im Anschluß an die Arbeiten der genannten Autoren zu überprüfen und zu 
präzisieren. Von ihnen war besonders WeILL bemüht, die allgemeinen Regeln aufzufinden, die sich aus der Verteilung der NK-Typen auf die systematischen Einheiten ergeben. Ausgangspunkt und zuverlässige Basis waren ihm jeweils die speziellen Untersuchungen, die ihn in die Lage versetzten, die in den höheren Gruppen vorkommenden NK-Typen vergleichend zusammenzustellen. Da die Zahl der Arten, deren

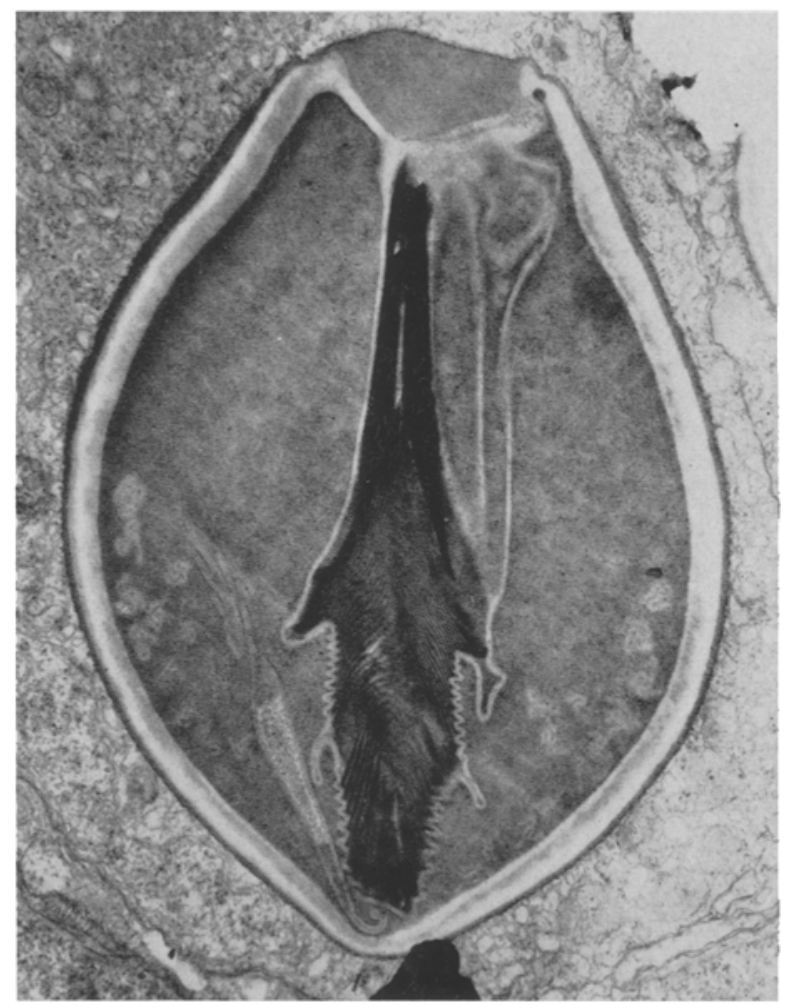

Abb. 22: Stenotele von Hydra spec. (Hydroida Hydrina), elektronenoptischer Längsschnitt durch die unentladene Kapsel, X 8500, Originalphoto von G. B. Chapman, New York

Nesselzellausstattung vollständig bekannt ist, immer noch als gering anzusehen ist, können derartige Untersuchungen nur vorläufigen Wert haben. Die bisherigen Ergebnisse sind jedoch bereits so interessant und wichtig, daß es lohnend erscheint, den Stand der Kenntnisse in einigen Ubersichten zusammenzufassen, soweit dies nach den Resultaten der mehrfach zitierten Autoren, vor allem wiederum von WEILL, möglich ist.

Die Grundlage für die Auswertung der vorliegenden Tatsachen ist ihre tabellarische Darstellung, wie sie im folgenden durchgeführt wird. Wie mehrfach angedeutet, gipfelt die Zielsetzung in der Frage, ob allgemein gültige Aussagen über die systematische und evolutionistische Bedeutung der Nesselzellausstattung möglich sind. Die Fragestellung lautet also: lassen sich die systematischen Einheiten auf Grund ihrer Nesselzellverhältnisse eindeutig kennzeichnen und einordnen; sie läßt sich für die verschiedenen systematischen Niveaus in mehrere Teilfragen aufgliedern. 


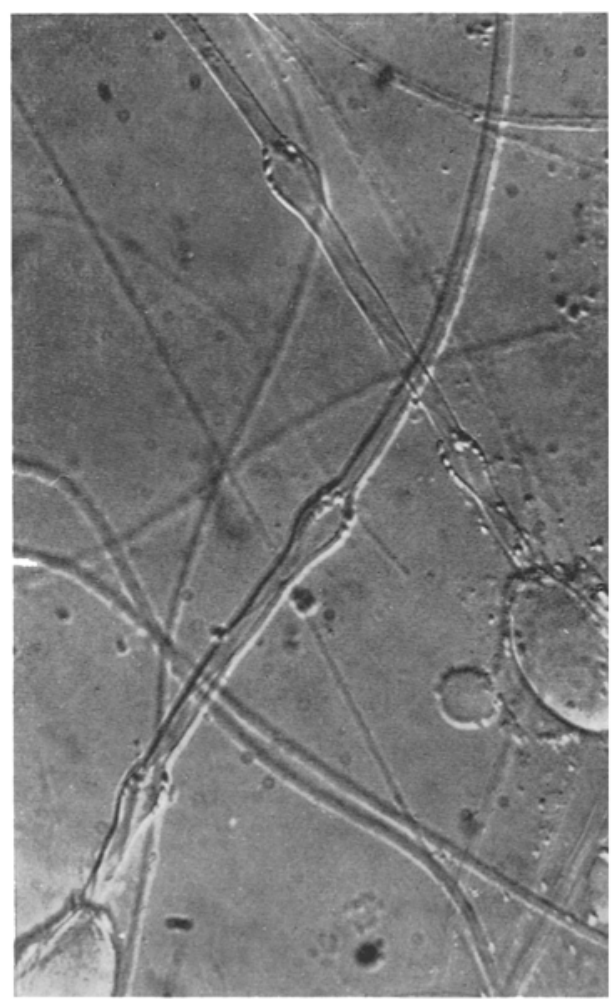

Abb. 23: Birhopaloiden von Apolemia uvaria (Siphonophora), entladene Kapseln, vergleidhe $\mathrm{Abb} .20 \mathrm{~b}$. Originalphoto von R. WeILl, Bordeaux, Interferentialphasenkontrast

1. Die systematischen Einheiten sind zu prüfen:

a) auf die Qualität und Quantität der bei ihnen überhaupt vorhandenen NK-Typen;

b) auf die Qualität und Quantität der einheitsspezifischen NK-Typen, das heißt derer, die nur in einer der auf dem betreffenden Niveau zum Vergleich stehenden Einheiten auftreten;

c) auf die Qualität und Quantität der den Gruppen gemeinsamen NK-Typen.

2. Es ist zu fragen, ob sich die systematischen Einheiten durch den Grad der Differenziertheit ihrer NK-Typen unterscheiden.

3. Im Anschluß an die Schlußfolgerungen, die sich aus der Beantwortung der Fragen 1 und 2 ergeben, muß untersucht werden, ob der Besitz spezieller Kapseltypen Aussagen für die Systematik der Gruppen gestattet.

\section{VORBEMERKUNGEN}

Die Analysen der qualitativen und quantitativen Verteilung sind in den Tabellen 2 bis 7 durchgeführt, in denen sämtliche NK-Typen auf die systematischen Einheiten der verschiedenen Niveaus aufgeteilt sind, für die ihr Vorkommen bislang 
signalisiert ist. Dabei werden nur die wirklich verschiedenen Kategorien getrennt aufgeführt, nicht aber Form-, Größen- oder Strukturvarietäten des gleichen Typs. Da weiterhin die homo- und heterotrichen mikrobasischen Eurytelen $(14,15)$ ebenso wie die telo-, mero- und holotrichen makrobasischen Eurytelen $(16,17,18)$ aus praktischen Gründen nicht getrennt behandelt zu werden brauchen, läßt sich die Zahl der verschiedenen Typen für unsere Vergleichszwecke von 20 auf 17 reduzieren.

WeILL (1934) hat in seinen Ubersichten in der gleichen Weise auch die homo- und heterotrichen Anisorhizen $(8,9)$ zusammengefaßt. Da beide Kategorien aber nicht nur nach der Bedornung, sondern auch nach der Form des Nesselapparates verschieden sind und außerdem auf dem Ordnungsniveau in verschiedenen Einheiten vorkommen, werden sie hier getrennt behandelt.

$\mathrm{Zu}$ berücksichtigen ist überdies, daß die Anthozoa wie erwähnt (siehe p. 12 ff.) über den weiteren, nur bei ihnen vorkommenden Typ der Spirocysten verfügen, der eigentlich in die vergleichende qualitative und quantitative Betrachtung einbezogen werden müßte. Im Anschluß an WerLL soll indes aus den schon erwähnten Gründen dieser Typ nicht generell berücksichtigt werden.

$\mathrm{Da}$ die eigenen Untersuchungen vorwiegend an Hydroiden ausgeführt wurden, kann die Behandlung der Tatsachenbasis für die Klasse Hydrozoa bis zu den Unterordnungen der Hydroida gebracht werden. Für die übrigen Klassen ist sie bis zu den Ordnungen durchgeführt; dabei zeigt sich, daß hier prinzipiell übereinstimmende Ergebnisse abgeleitet werden können. Die Analyse auf die niedrigeren Niveaus der Familien, Gattungen und Arten auszudehnen, würde zu weit führen und ist hier nicht beabsichtigt.

Den tabellarischen Obersichten wird das bisher gültige System zugrunde gelegt, das in einigen Punkten modifiziert ist.

Stamm Cnidaria, Klassen: Hydrozoa, Scyphozoa, Anthozoa.

Hydrozoa Ordnungen:

Scyphozoa Ordnungen:

Hydroida, Trachylida, Siphonophora.

Stauromedusae, Cubomedusae, Coronatae, Semaeostomeae, Rhizostomeae.

Anthozoa Unterklassen: Hexacorallia, Octocorallia.

Hexacorallia Ordnungen: Actiniaria, Ceriantharia, Madreporaria, Antipatharia, Zoantharia.

Hydroida Unterordnungen: Athecata, Hydrina, Thecata, Limnohydrina, Halammohydrina.

Mit der Einordnung der Hexacorallia vor den Octocorallia folge ich PAx (1940), KaEsTNER (1965) und besonders HaDžr (1963, p. 231 f.), der ausführlich die systematische Situation erörtert und die Gründe angibt, die für die Stellung der Hexacorallia an der Basis der Anthozoa und die Reihenfolge der Ordnungen sprechen.

Die Gliederung der Hydroida in die 5 Unterordnungen entspricht meines Erachtens am besten den tatsächlichen Gegebenheiten. In der ausländischen Literatur werden die hier als Unterordnungen aufgeführten Einheiten vielfach in den Rang einer Ordnung erhoben (vgl. Russel. 1953, KRAMP 1961 für die Medusengeneration), womit aber ihre enge Zusammengehörigkeit nicht ausreichend berücksichtigt wird. Mit der 
Abtrennung der Hydrina von den Athecata schließe ich mich Naumov (1960, nach THiel 1962) an, der die Gruppe als Hydrida unter den Ordnungen anführt. Die Limnohydrina hat KRAMP (1938 und später als Limnomedusae bezeichnet) als eigene Unterordnung der Hydroida eingeführt; die zugehörigen Familien sind die Olindiidae (die früher zu den Trachymedusae gestellt wurden), ferner die Limnocnididae und Proboscidactylidae. KRAMP hat im wesentlichen stets nur die Medusengeneration berücksichtigt, woraus sich seine Namengebung erklärt. Im Interesse einer einheitlichen Kennzeichnung ist der hier eingeführte Name Limnohydrina, der gleichzeitig das Vorhandensein einer echten Polypengeneration zum Ausdruck bringen soll, vorzuziehen. Bezüglich der Einordnung der Halammohydrina als Unterordnung der Hydroida vergleiche WERNER (1965). Die früher in den Hydrocorallidae vereinigten, neuerdings aber getrennten Familien Milleporidae und Stylasteridae werden zu den Athecata gestellt (vgl. Stechow 1923, KaEstner 1965). Das wird deswegen erwähnt, weil sie von Weill (1934) als eine Einheit neben die Athecata ("Gymnoblastes") und Thecata ("Calyptoblastes“) gestellt werden.

HADŽI $(1958,1963)$ hat die Auffassung vertreten, daß die Reihenfolge der Klassen umgekehrt werden müsse, da die Anthozoa als niedrigste Gruppe von den Turbellaria abzuleiten seien. $\mathrm{Zu}$ dieser Hypothese wird im Anschluß an die Erörterung der Ergebnisse kurz Stellung zu nehmen sein, soweit dies unter dem Aspekt der Nesselzellausstattung möglich ist.

\section{DIE BEI DEN CNIDARIA VORHANDENEN KAPSELTYPEN}

\section{Die allgemeine qualitative und quantitative Verteilung}

Welche NK-Typen beiden systematischen Einheiten der verschiedenen Niveaus $\ddot{u} \mathrm{berhaupt}$ vorkommen, geht aus den Tabellen 2 bis 7 hervor, auf die zur Beantwortung dieser ersten Teilfrage verwiesen werden kann. Wenn man an Hand der tabellarischen Übersichten die Gruppen auf ihre Nesselkapseln vergleicht, so ergibt sich ohne weiteres das folgende Resultat, das für sämtliche Niveaus gilt: keine Gruppe besitzt ein Muster von verschiedenen NK-Typen, die in ihrer Gesamtheit ausschließlich bei ihr vorkommen; vielmehr hat jede Gruppe stets auch Typen mit anderen Einheiten gemeinsam. Damit läßt sich keine systematische Gruppe durch die Art und den Gesamtbestand der verschiedenen NK-Typen eindeutig positiv charakterisieren. Wir gelangen damit zum ersten allgemeinen Ergebnis, daß die Nesselzellausstattung nach der qualitativen Verteilung kein systematisches Merkmal von allgemeiner, absoluter Gültigkeit darstellt.

Wie verhält es sich weiterhin mit der Anzahl der bei den Gruppen vorhandenen NK-Typen? Zur besseren Ubersicht sind die in den Tabellen 2 bis 7 enthaltenen quantitativen Verhältnisse in der Tabelle 8 nochmals zusammengefaßt, wobei die Einheiten mit dem quantitativen Maximum auf dem betreffenden Niveau durch Fettdruck hervorgehoben sind. Wäre die quantitative Verteilung von systematischer Bedeutung, so sollte man erwarten, daß die niederen systematischen Einheiten eine geringere Zah1 
Nesselkapseln der Cnidaria

Tabelle 2

Die Nesselkapseln der Cnidaria

\begin{tabular}{|c|c|c|c|}
\hline Typen/Klassen & Hydrozoa & Scyphozoa & Anthozoa \\
\hline Anacrophoren (1) & 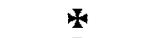 & - & - \\
\hline Acrophoren (2) & 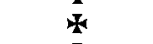 & - & - \\
\hline Desmonemen (3) & ( & - & - \\
\hline atriche Haplonemen (4) & + & + & + \\
\hline basitriche Haplonemen (5) & + & - & + \\
\hline merotriche Haplonemen (6) & $\Psi$ & - & - \\
\hline holotriche Haplonemen (7) & + & + & + \\
\hline homotriche Anisorhizen (8) & 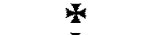 & - & - \\
\hline heterotriche Anisorhizen (9) & 4 & - & - \\
\hline mikrobasische Mastigophoren (10) & + & - & + \\
\hline makrobasische Mastigophoren (11) & * & - & - \\
\hline mikrobasische Amastigophoren (12) & - & - & * \\
\hline makrobasische Amastigophoren (13) & - & - & + \\
\hline mikrobasische Eurytelen (14) (15) & \pm & + & - \\
\hline makrobasische Eurytelen (16) (17) (18) & w & - & - \\
\hline Stenotelen (19) & 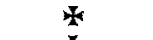 & - & - \\
\hline Birhopaloiden (20) & 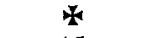 & - & - \\
\hline Gesamtzabl der verschiedenen Typen & 15 & 3 & 6 \\
\hline Gesamtzabl der klasseneigenen Typen & 10 & 0 & 2 \\
\hline
\end{tabular}

Anmerkung zu den Tabellen 2 bis 7: Die in den Tabellen 2 bis 7 enthaltenen Angaben über die qualitative und quantitative Verteilung der Kapseltypen sind entnommen: für die Hydrozoa: Weill 1934, vgl. besonders p. 526, Weill 1964, Russell 1938, 1939, 1940, vgl. 1953, Hand 1954 a, Picard 1955 a, b, Bourlzon 1957; Itô \& Inoue 1962; für die Scyphozoa: Weill 1934, vgl. besonders p. 548, Papenfuss 1936; für die Anthozoa: Weile 1934, vgl. besonders p. 592 f., 616, 628, Tischbierek 1936, Carlgren 1940, 1945, Hand 1954 b, 1955 a, b. In den Tabellen 2 bis 7 bedeuten: + vorhanden, - nicht vorhanden, einheitseigener Typ.

Tabelle 3

Die Nesselkapseln der Hýdrozoa

\begin{tabular}{|c|c|c|c|}
\hline Typen/Ordnungen & Hydroida & Trachylida & Siphonophora \\
\hline Anacrophoren (1) & - & - & + \\
\hline Acrophoren (2) & - & - & * \\
\hline Desmonemen (3) & + & - & + \\
\hline atriche Haplonemen (4) & + & + & + \\
\hline basitriche Haplonemen (5) & 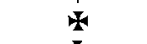 & - & - \\
\hline merotriche Haplonemen (6) & 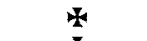 & - & - \\
\hline holotriche Haplonemen (7) & 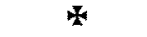 & - & - \\
\hline homotriche Anisorhizen (8) & - & - & * \\
\hline heterotriche Anisorhizen (9) & 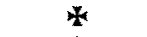 & - & - \\
\hline mikrobasische Mastigophoren (10) & + & - & + \\
\hline makrobasische Mastigophoren (11) & 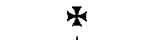 & - & - \\
\hline mikrobasische Eurytelen (14) (15) & + & + & - \\
\hline makrobasische Eurytelen (16) (17) (18) & + & - & + \\
\hline Stenotelen (19) & + & + & + \\
\hline Birhopaloiden (20) & - & - & 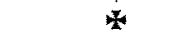 \\
\hline Gesamtzabl der verschiedenen Typen & 11 & 3 & 9 \\
\hline Gesamtzabl der ordnungseigenen Typen & 5 & 0 & 4 \\
\hline
\end{tabular}


Tabelle 4

Die Nesselkapseln der Scyphozoa

Für die Cubomedusae liegen noch keine Angaben vor

\begin{tabular}{|lcccc|}
\hline Typen/Ordnungen & $\begin{array}{c}\text { Stauro- } \\
\text { medusae }\end{array}$ & $\begin{array}{c}\text { Coro-1 } \\
\text { natae }\end{array}$ & $\begin{array}{c}\text { Semaeo- } \\
\text { stomeae }\end{array}$ & $\begin{array}{c}\text { Rhizo- } \\
\text { stomeae }\end{array}$ \\
\hline atriche Haplonemen (4) & + & - & + & + \\
holotriche Haplonemen (7) & + & + & + & + \\
mikrobasische Eurytelen (15) & + & + & + & + \\
Gesamtzabl der verschiedenen Typen & 2 & 2 & 3 & 2 \\
Gesamtzabl der ordnungseigenen Typen & 0 & 0 & 0 & 0 \\
1 Nach eigenen unveröfentlichten Beobachtungen an Stephanoscyphus-Polypen und \\
den von ihnen durch Strobilation erzeugten Medusen, die den Gattungen Nausitboe und \\
Atorella angehören. Das Material konnte lebend aus dem Indischen Ozean mitgebracht \\
und in Kultur genommen werden.
\end{tabular}

Tabelle 5

Die Nesselkapseln der Anthozoa

\begin{tabular}{|c|c|c|}
\hline Typen/Unterklassen & Hexacorallia & Octocorallia \\
\hline atriche Haplonemen (4) & + & + \\
\hline basitriche Haplonemen (5) & * & - \\
\hline holotriche Haplonemen (7) & * & - \\
\hline mikrobasische Mastigophoren ( 10 & 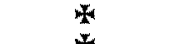 & - \\
\hline mikrobasische Amastigophoren (12) & * & - \\
\hline makrobasische Amastigophoren (13) & t & - \\
\hline (Spirocysten) (21) & (4) & - \\
\hline Gesamtzahl der verschiedenen Typen & $6(7)$ & 1 \\
\hline Gesamtzahl der unterklasseneigenen Typen & $5(6)$ & 0 \\
\hline
\end{tabular}

Tabelle 6

Dir: Nesselkapseln der Hexacorallia

\begin{tabular}{|lccccc|}
\hline Typen/Ordnungen & $\begin{array}{c}\text { Acti- } \\
\text { niaria }\end{array}$ & $\begin{array}{c}\text { Cerian- } \\
\text { tharia }\end{array}$ & $\begin{array}{c}\text { Madre- } \\
\text { poraria }\end{array}$ & $\begin{array}{c}\text { Anti- } \\
\text { patharia }\end{array}$ & $\begin{array}{c}\text { Zoan- } \\
\text { tharia }\end{array}$ \\
\hline $\begin{array}{l}\text { atriche Haplonemen (4) } \\
\text { basitriche Haplonemen (5) }\end{array}$ & + & + & - & - & - \\
holotriche Haplonemen (7) & + & + & + & + & - \\
mikrobasische Mastigophoren (10 & + & + & + & + & + \\
mikrobasische Amastigophoren (12) & + & - & - & - & - \\
makrobasische Amastigophoren (13) & $(+)$ & $(+)$ & $(+)$ & $(+)$ & $(+)$ \\
(Spirocysten) (21) & $6(7)$ & $3(4)$ & $2(3)$ & $3(4)$ & $2(3)$ \\
Gesamtzabl der verschiedenen Typen & 2 & 0 & 0 & 0 & 0 \\
Gesamtzahl der ordnungseigenen & & & & & \\
Typen & & & & & \\
\hline
\end{tabular}


Tabelle 7

Die Nesselkapseln der Hydroida

\begin{tabular}{|c|c|c|c|c|c|}
\hline Typen/Unterordnungen & $\begin{array}{l}\text { Athe- } \\
\text { cata }\end{array}$ & $\begin{array}{c}\mathrm{Hy-} \\
\text { drina }\end{array}$ & $\begin{array}{l}\text { The- } \\
\text { cata }\end{array}$ & $\begin{array}{l}\text { Limno- } \\
\text { hydrina }\end{array}$ & $\begin{array}{c}\text { Halammo- } \\
\text { hydrina }\end{array}$ \\
\hline Desmonemen (3) & + & + & - & + & + \\
\hline atriche Haplonemen (4) & + & + & + & + & + \\
\hline basitriche Haplonemen (5) & + & - & + & + & - \\
\hline merotriche Haplonemen (6) & - & - & * & - & - \\
\hline holotriche Haplonemen (7) & - & * & - & - & - \\
\hline heterotriche Anisorhizen (9) & * & - & - & - & - \\
\hline mikrobasische Mastigophoren (10) & + & - & + & -1 & - \\
\hline makrobasische Mastigophoren (11) & * & - & - & - & - \\
\hline mikrobasische Eurytelen (14) (15) & + & - & + & +1 & - \\
\hline makrobasische Eurytelen (16) (17) (18) & + & - & - & + & - \\
\hline Stenotelen (19) & + & + & - & - & + \\
\hline Gesamtzabl der verschiedenen Typen & 9 & 4 & 5 & 5 & 3 \\
\hline $\begin{array}{l}\text { Gesamtzabl der unterordnungseigenen } \\
\text { Typen } *\end{array}$ & 2 & 1 & 1 & 0 & 0 \\
\hline
\end{tabular}

verschiedener Nesselkapseltypen aufwiesen als die höheren. Die vergleichende Prüfung anhand der Tabelle 8 zeigt jedoch überraschenderweise, daß das nicht der Fall ist, daß vielmehr auf allen Niveaus die im geltenden System an der Basis stehenden Gruppen über die größte Zahl an verschiedenen NK-Typen verfügen. Das Resultat gilt von den Klassen bis zu den Unterordnungen. Eine Ausnahme machen nur die Ordnungen der Scyphozoa; doch erklärt sie sich hier aus der geringen $\mathrm{Zahl}$ überhaupt vorhandener NK-Typen, so daß quantitative Aussagen schon aus diesem Grunde wenig sinnvoll sind. Andererseits geht aus den Tabellen hervor, daß nicht umgekehrt die im System höchsten Einheiten durchweg die geringste Zahl von verschiedenen NK-Typen besitzen. Vielmehr haben etwa die Anthozoa mehr Typen als die Scyphozoa, die Siphonophora mehr als die Trachylida. Nach allem besteht keine eindeutige Relation zwischen der Stellung einer Gruppe im System und der Zahl der bei ihr vorkommenden NK-Typen. Damit ist auch die quantitative Verteilung kein Merkmal von allgemeiner systematischer Bedeutung.

Aus der Verteilung a uf die systematischen Gruppen läßt sich als weiteres Resultat ablesen, daß die meisten über ein heitsspe z if is che NK-T y p en verfügen. Die Tabelle 2 demonstriert zum Beispiel, daß die Astomocniden auf die Klasse Hydrozoa beschränkt sind, während die Stomocniden mit Haplo- und Heteronemen auf alle drei Klassen verteilt sind. Allerdings gibt es bei den Heteronemen 2 Kategorien, die nur bei den Anthozoa, sowie 4, die nur bei den Hydrozoa gefunden werden. Die Existenz derartiger einheitsspezifischer NK-Typen ermöglicht offensichtlich eine beschränkte Charakterisierung der betreffenden systematischen Gruppen; denn ihr Nachweis gestattet sofort die Zuordnung zu der jeweiligen Einheit der verschiedenen Niveaus. Findet man beispielsweise bei einem Cnidarier 
Desmonemen, so resultiert aus Tabelle 2 sofort, daß es sich nur um ein Hydrozoon handeln kann; andererseits kommen Desmonemen laut Tabelle 3 sowohl bei den Hydroida wie bei den Siphonophora vor, so daß hier die Zuordnung nicht mehr eindeutig ist. Es kann mithin ein NK-Typ auf einem systematischen Niveau einheitsspezifisch sein, auf dem nächst niederen aber nicht mehr. Wie die Tabellen erkennen lassen, sind einige NK-Typen gleichzeitig auf mehreren Niveaus einheitsspezifisch; so die Ana- und Acrophoren (1,2), die homotrichen Anisorhizen (8) und die Birhopaloiden (20) für die Hydrozoa Síphonophora, die heterotrichen Anisorhizen (9), die makrobasischen Mastigophoren (11) für die Hydrozoa Hydroida Athecata, die merotrichen Haplonemen (6) für die Hydrozoa Hydroida Thecata, die mikro- und makrobasischen Amastigophoren $(12,13)$ für die Anthozoa Hexacorallia Actiniaria, so daß hier die Zugehörigkeit einer Form zu den Ordnungen beziehungsweise zu den Unterordnungen auf Grund der Nesselkapseln klar ersichtlich ist. Durch die einheitsspezifischen NK-Typen ist damit eine begrenzte, relative Charakterisierung der Gruppen auf den systematischen Niveaus möglich. Dieses Ergebnis steht in enger Beziehung zu dem systematischen Wert, den die Nesselzellausstattung durch die speziellen Kapseltypen hat, worauf später noch näher einzugehen ist. Hinsichtlich der quantitativen Verteilung der einheitsspezifischen NK-Typen (Tab. 8, Spalte 2) ist ein weiteres bemerkenswertes Resultat, daß die Einheiten mit dem absoluten quantitativen Maximum an verschiedenen NK-Typen (Spalte 1) gleichzeitig auch diejenigen sind, auf die das Maximum an einheitsspezifischen NK-Typen entfällt.

Nach der allgemeinen qualitativen und quantitativen Verteilung bleiben noch die NK-Typen zu berücksichtigen, die auf den Niveaus allen Gruppen gemein sam sind. Dabei stellt sich heraus, daß den drei Klassen (Tab. 2) zwei Typen gemeinsam sind, nämlich die atrichen und holotrichen Haplonemen $(4,7)$. Das ist deswegen von Interesse, weil es sich bei den Haplonemen um die morphologisch einfachen Nesselkapseln handelt, die evolutiv an der Basis stehen. Aus Tabelle 8, Spalte 3 ist abzulesen, daß mit einer Ausnahme in allen Niveaus die Haplonemen die den Gruppen gemeinsamen NK-Typen stellen, und zwar entweder ausschließlich oder zusammen mit 1 Typ von Heteronemen. Hinsichtlich der quantitativen Verteilung gestattet die geringe Zahl von gemeinsamen Typen kaum eine sichere Aussage; immerhin ist bemerkenswert, daß auf dem höchsten Niveau der Klassen wie auch bei einem Teil der Ordnungen zwei gemeinsame Typen vorkommen, bei dem Rest der Ordnungen und bei den geprüften Unterordnungen aber nur ein Typ gemeinsam ist. Bei den Ordnungen der Hydrozoa ist weiterhin zu beachten, daß neben einem einfachen Typ der Haplonemen auch ein hochdifferenzierter Typ, nämlich der der Stenotelen (19) aus der Gruppe der Heteronemen gemeinsam ist. Wir berühren $\mathrm{da}$ mit erneut die Frage nach der Bedeutung der speziellen Kapseltypen, die später erörtert wird. Es sei schon jetzt darauf hingewiesen, daß der gemeinsame Besitz der Stenotelen in diesem speziellen Fall $z$ weifellos ein Ausdruck der verwandtschaftlichen Zusammengehörigkeit ist.

WeILl hat auf Grund der morphologischen Merkmale eine evolutive Reihe der Stomocniden aufstellen können, an deren Basis die Haplonemen stehen; aus ihnen sind die höher differenzierten Typen der Heteronemen entstanden zu denken (vgl. WeIlx 1934, p. 98 ff., Fig. 86, p. 99, CARLGRen 1940, p. 52 ff.). Dieses zunächst nur 


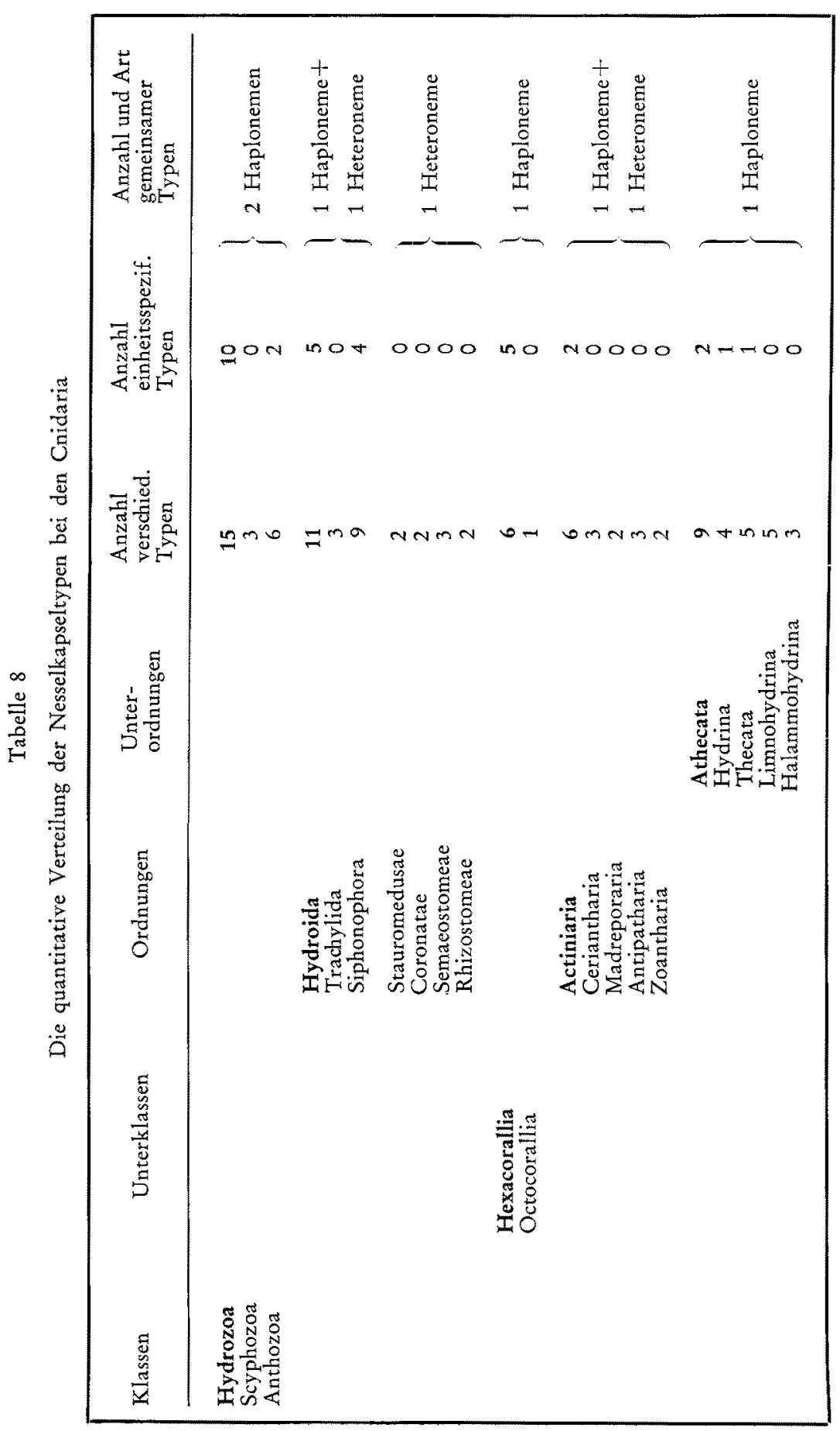


aus der Struktur abgeleitete Ergebnis steht in guter Übereinstimmung mit der Verteilung der Kategorien auf den geprüften systematischen Niveaus, insofern als es eben die Haplonemen sind, die den meisten Gruppen gemeinsam sind. Das legt den weiteren Schluß nahe, daß die Haplonemen bei den gemeinsamen Vorfahren der Vertreter aller drei Klassen vorhanden gewesen sind und führt schließlich zu der wohlbegründeten Vermutung, daß sich die Entwicklung von Haplo- zu Heteronemen an mehreren Stellen im System und unabhängig vollzogen hat (vgl. KüHN 1915, p. 442, WeILL 1934, p. 648 f.).

\section{Die Differenzierungshöhe der Kapseltypen}

Das Problem der strukturellen Qualität, auf das wir bereits bei den Betrachtungen über die Verteilung der einheitsspezifischen und gemeinsamen NK-Typen gestoßen sind, führt weiter zur Prüfung der konkreten Frage, ob zwischen den Einheiten quantitative Unterschiede im Anteil einfacher oder komplizierter NK-Typen nachweisbar sind. Für eine derartige Untersuchung sind weniger die Astomocniden als vielmehr die Stomocniden geeignet, eben weil sie, wie erwähnt, eine evolutive Reihe darstellen, in der die Haplonemen die einfachen, die Heteronemen aber die höher differenzierten Typen repräsentieren. Für die Beantwortung unserer Frage brauchen daher alle Einheiten lediglich auf den Anteil an Haplonemen und Heteronemen verglichen zu werden. Für unsere Zwecke genügt es, nur diese beiden Gruppen heranzuziehen, und es erübrigt sich, etwa bei den Heteronemen weiter zwischen den weniger und höher differenzierten Typen zu unterscheiden.

Die Tabelle 9 zeigt zunächst, daß tatsächlich Unterschiede zwischen den Einheiten der verschiedenen Niveaus bestehen. Wäre allerdings die in unserem Sinne verstandene allgemeine Differenzierungshöhe ein Merkmal von systematischer Bedeutung, so müßten die nach dem System niedrigen Gruppen mehr einfache, weniger komplizierte Typen aufweisen, das heißt der Quotient Haplonemen :Heteronemen ( $\mathrm{Ha}: \mathrm{He}$ ) sollte größer als 1 sein. Wenn wir die tatsächlichen Verhältnisse anhand der Tabelle 9 überblicken und vor allem bei den niedrigen Einheiten nachschauen, so zeigt sich sofort, $\mathrm{da}$ diese theoretische Forderung auf keinem Niveau erfüllt ist. Wir sehen vielmehr, daß bei den jeweils niedrigsten Einheiten aller Niveaus genau das umgekehrte der Fall ist; der Quotient $\mathrm{Ha}: \mathrm{He}$ ist entweder kleiner oder gleich 1, weil die höher differenzierten Kapseltypen einen großen Anteil von mindestens 50\% oder darüber stellen.

Aber auch der umgekehrte Fall, in dem die höheren Einheiten mehr Haplonemen besitzen sollten als Heteronemen, ist nicht durchgehend erfüllt; insbesondere triff er nicht zu auf der Klassenebene bei den Anthozoa, bei denen der Quotient $\mathrm{Ha}$ : He wie bei den Hydrozoa 1 ist.

Andererseits wäre theoretisch zu fordern, daß die nach dem System höheren Einheiten einen größeren Anteil von Heteronemen ihr Eigen nennen, so daß bei ihnen die Relation erfüllt sein sollte $\mathrm{Ha}: \mathrm{He}<1$. Nach Tabelle 9 ist diese Forderung nur bei den Trachylida und Siphonophora erfüllt, nicht aber bei den übrigen, das heißt der Mehrzahl der höheren Gruppen. Wir gelangen damit zu dem eindeutigen Resultat, 


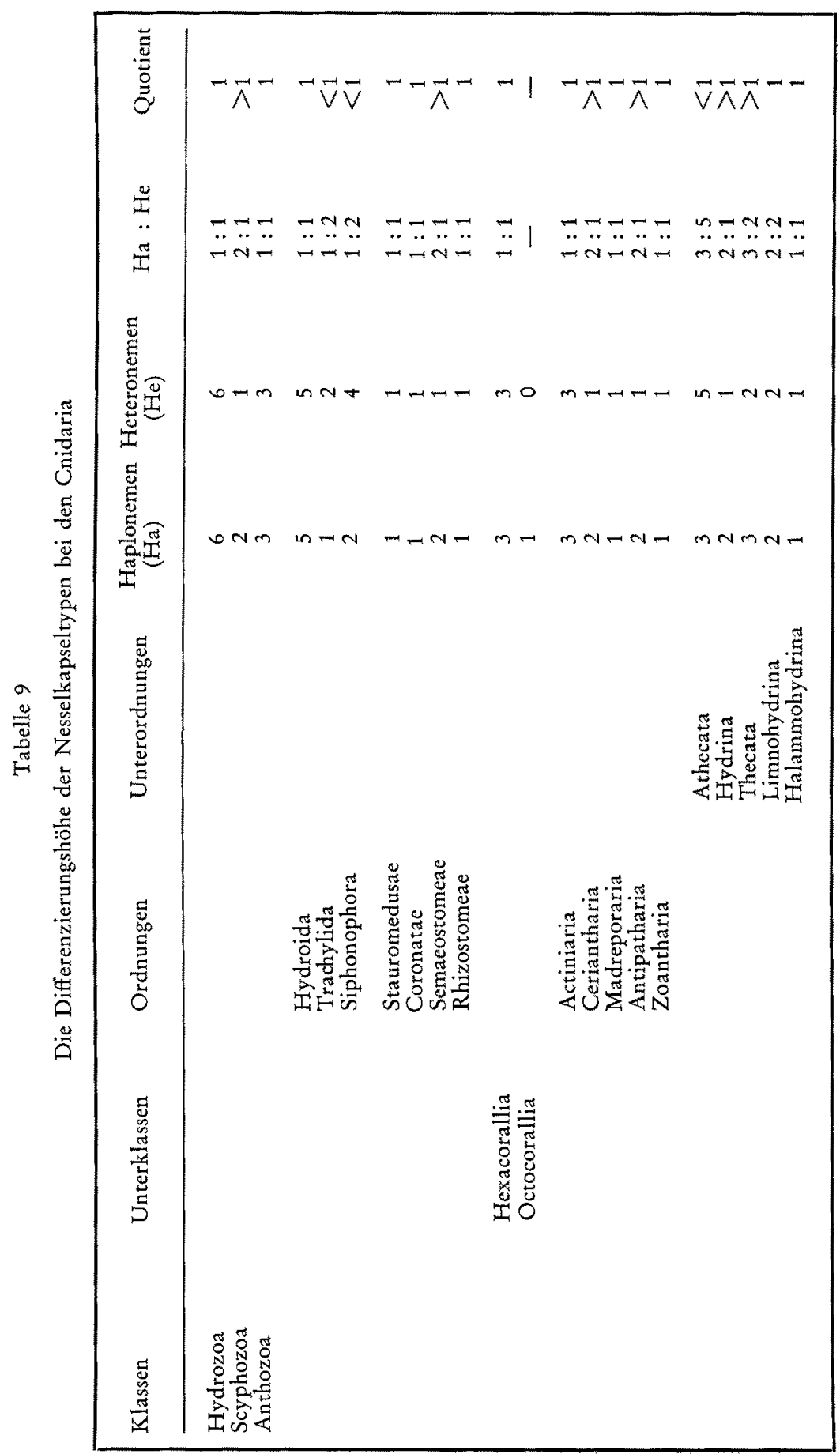


daß die Differenzierungshöhe kein Merkmal von allgemeiner systematischer Bedeutung ist.

\section{Die Bedeutung der speziellen Kapseltypen}

Nach allem bisher Gesagten gestatten die Schlußfolgerungen hinsichtlich der systematischen und damit der evolutionistischen Bedeutung der Nesselzellverhältnisse vorerst nur eine überwiegend negative Beurteilung. Erschöpfen sich die Aussagemöglichkeiten in dieser negativen Bewertung? Prüfen wir den dritten wesentlichen Aspekt, auf den wir bereits mehrfach gestoßen sind und der mit dem Besitz spezieller Kapseltypen gegeben ist; er betrifft daher die spezielle Qualität der bei den Einheiten vorhandenen Kapseltypen.

Wir sahen bereits, daß die einheitsspezifischen Kapseltypen auf den geprüften Niveaus eine allerdings begrenzte, relative Charakterisierung zulassen (siehe p. $21 \mathrm{f}$ ). Wenn wir die Verteilung der Kapseltypen nach den Tabellen 2 bis 7 unter dem Aspekt ihrer speziellen Qualität und wenn wir daraufhin die Gruppen auf den Niveaus vergleichend betrachten, gelangen wir zu weiteren Aussagen, die aus konkreten Sachverhalten resultieren. So geht aus der Tabelle 2 hervor, daß die Scyphozoa nur NKTypen besitzen, die auch bei den Hydrozoa vorkommen. Daraus kann mit Werll (1934, p. 632 f.) geschlossen werden, daß die Scyphozoa nach ihrer Nesselzellausstattung den Hydrozoa näherstehen als den Anthozoa, die erstens mehr verschiedene Kapseltypen besitzen und zweitens durch den Besitz einheitspezifischer Typen sich auch von den Hydrozoa weiter entfernt haben. Ahnliche Verhälnisse lassen sich auch auf den anderen Niveaus nachweisen. So haben bei den Hydrozoa die Trachylida nur Nesselkapseln, die wir auch bei den Hydroida wiederfinden. Für die Trachylida dürfte daher eine entsprechende Schlußfolgerung zulässig sein, nämlich daß sie sich nach ihrer Nesselzellausstattung enger an die Hydroida anschließen als an die Siphonophora, die sich nach der relativ hohen Anzahl einheitsspezifischer NK-Typen unabhängig weiter entwickelt haben. Andererseits haben die Siphonophora mit den Hydroida zwei so hoch spezialisierte Kapseltypen wie die makrobasischen Eurytelen $(16,17,18)$ und Stenotelen (19) gemeinsam, daß an dem evolutionistischen Zusammenhang dieser beiden Ordnungen nicht zu zweifeln ist, das heißt, daß sich die Siphonophora von den Hydroida herleiten (siehe p. 28). Wenn wir schließlich den spezialisierten Typ der Stenotelen (19) bei allen drei Ordnungen der Hydrozoa antreffen, so ist dieses Merkmal zweifellos als Ausdruck der Zusammengehörigkeit dieser Gruppen zu werten, worauf schon kurz hingewiesen wurde.

Bei den Scyphozoa scheinen die Aussagemöglichkeiten über das gegenseitige Verhältnis der Ordnungen auf Grund spezieller Kapseltypen nicht so eindeutig zu sein, was wohl mit der geringen Zahl überhaupt vorhandener Typen zu erklären ist.

Bei den Anthozoa fällt sofort die scharfe Trennungslinie auf, die in den Nesselzellverhältnissen zwischen den Hexa- und Octocorallia besteht, weil bei den Formen aus der letzteren Unterklasse nur ein einziger Kapseltyp gefunden wird. Eine plausible Erklärung für diese Tatsache kann bislang offenbar nicht gegeben werden; in jedem Fall fehlen konkrete Hinweise dafür, daß die Armut an Nesselkapseltypen bei den 
Octocorallia als Ausdruck einer regressiven Entwicklung betrachtet werden müßte. Vielmehr ist die positive Tatsache hervorzuheben, daß es wiederum der spezielle Typ der atrichen Haplonemen (4) ist, der den beiden Unterklassen gemeinsam ist, eine weitere Stütze für die Auffassung, daß es sich um den morphologisch und evolutiv einfachsten Typ handelt. Die Trennungslinie zwischen den beiden Unterklassen wird noch dadurch verschärt, daß nur die Hexacorallia mit sämtlichen Ordnungen den speziellen Typ der Spirocysten besitzen (vgl. p. 20, Tab. 6). Innerhalb dieser Unterklasse heben sich die Actiniaria durch die große Zahl der Kapseltypen sowie durch den Besitz der einheitsspezifischen Amastigophoren $(12,13)$ heraus, so daß die Ordnungen der Ceriantharia, Madreporaria, Antipatharia und Zoantharia nach dem negativen Merkmal des Fehlens dieser Typen eine gemeinsame Gruppe bilden. Im übrigen sind die Unterschiede zwischen diesen letzteren 4 Ordnungen offenbar nicht so ausgeprägt, daß ihre Nesselzellausstattung evolutionistische Entwicklungslinien besonders deutlich machen könnte. Daß gleichwohl die Nesselzellverhältnisse auf Grund der speziellen Kapseltypen für die Systematik der Hexacorallia von Bedeutung sind, hat CARLGREN 1940 ausführlich erörtert.

Auf dem systematischen Niveau der Unterordnungen sind bei den Hydroida entsprechende Beziehungen erkennbar. Laut Tabelle 7 schließen sich die Hydrina und Halammohydrina durch den Besitz der Desmonemen (3) und Stenotelen (19) an die Athecata an, während für die Limnohydrina eine Verbindung zu den Athecata durch die Desmonemen (3) und mikro- und makrobasischen Eurytelen $(15,18)$ besteht. Hier gibt die Nesselzellausstattung augenscheinliche Hinweise auf die evolutiven Entwicklungslinien. Für den bei allen Unterordnungen der Hydroida gemeinsamen Typ der atrichen Haplonemen (4) muß ergänzend bemerkt werden, daß er auf der nächst niederen Stufe der Familien die verbindende Rolle nicht mehr spielt. Das gilt zum Beispiel für zahlreiche Familien der Sectio Filifera der Athecata, denen atriche Haplonemen fehlen.

Bei allen diesen Beispielen ließen sich verwandtschaftliche Beziehungen und Verbindungslinien zwischen den Einheiten auf Grund ihrer Nesselzellverhältnisse aufzeigen, worin sich die Bedeutung der speziellen Qualität der Kapseltypen für die Systematik und Evolution manifestiert. Es ist dieser besondere Aspekt, unter dem die Schlußfolgerungen von WErLL allgemeine Gültigkeit haben: „... le cnidome apporte toujours des renseignements taxonomiques extrêmement précieux et précis. Lorsque les documents anatomiques et embryologiques sont clairs, non équivoques et bien concordants, le cnidome confirme toujours leur conclusion; lorsque ces documents sont équivoques, discordants, et permettent deux ou plusieurs interprétations différentes, ce dilemme est toujours éclairci, harmonieusement et logiquement, par l'étude du cnidome." (1935, p. 75 f.) $\mathrm{Zu}$ prinzipiell übereinstimmenden Ergebnissen sind auch die übrigen Autoren gekommen, welche die Frage der systematischen Bedeutung der Nesselverhältnisse diskutiert haben, so yor allem CARLgren (1940, 1945) und Russell (1953).

Wir können nach allem die Untersuchung des systematischen Wertes der Nesselzellausstattung dahin zusammenfassen, daß positive Aussagen durch den Besitz der speziellen Kapseltypen möglich sind, die das nach morphologischen Merkmalen aufgebaute System stützen und bestätigen. 
Die Nesselzellverhältnisse können also in der gekennzeichneten Weise neben den vergleichend-morphologischen und entwicklungsgeschichtlichen Merkmalen zum Aufbau eines natürlichen Systems Verwendung finden. Wenn wir insbesondere die Fälle betrachten, in denen sich auf Grund der Verteilung der speziellen Kapseltypen Einheiten zu natürlichen Gruppen zusammenfassen lassen, so stehen die so erzielten Ergebnisse in Ubereinstimmung mit dem System. Um die Situation nochmals klarzustellen, sei ein bereits gebrauchtes Beispiel wiederholt: Wenn wir alle Species, die über Stenotelen verfügen, lediglich nach diesem Merkmal gruppieren und die systematische Zugehörigkeit prüfen, so stellt sich heraus, daß wir es ausschließlich mit Hydrozoa zu tun haben. Andererseits sind die mit speziellen Kapseltypen gegebenen Merkmale relativ und nur auf bestimmten Niveaus gültig. Speziell bei den Hydrozoa kommen in allen Klassen und allen Ordnungen Stenotelen vor, aber nicht in allen Unterordnungen, Familien, Gattungen, Species.

Darüber hinaus kann nad WEILL (siehe obiges Zitat) der Besitz spezieller Kapseltypen in zweifelhaften Fällen, bei denen die systematische Stellung einer Species oder einer Gruppe ebenso wie die phylogenetische Ableitung auf Grund der morphologischen Daten unklar bleibt, positive Hinweise für die Einordnung geben. Dafür seien aus der Literatur einige demonstrative Beispiele angeführt.

PICARD (1955b) hat entdeckt, daß die Medusoide von Velella (Siphonophora Chondrophoridae) den in den übrigen Gruppen der Siphonophora fehlenden Kapseltyp der makrobasischen Eurytelen $(16,17,18)$ besitzen. Er folgert daraus, daß sich die Chondrophoridae von der Familie Zancleidae (syn. Pteronematidae) der Hydroida Athecata ableiten, die in Zanclea costata die Species mit dem gleichen Kapseltyp besitzen. Da die Medusoide von Velella auch morphologisch den Medusen von Zanclea ähnlich sind, erscheint die Schlußfolgerung von PICARD wohlbegründet. Daß dieser Autor (1957) die Velellidae (Velella, Porpita) auf Grund dieses Befundes allerdings aus den Siphonophora vollständig herausnimmt und als Familie in die Athecata einreiht (vgl. BrincKMANn 1964), erscheint eine zu weitgehende Konsequenz, auch wenn an der evolutionistischen Entwidklungslinie von den Zancleidae zu Velella nach dem Vorhandensein des erwähnten speziellen Kapseltyps nicht zu zweifeln ist (vgl. dazu auch TotTon \& MackiE 1960).

Ein weiteres Beispiel hat Rees (1957a) gegeben. Die Familie Moerisiidae wird meist zu den Hydroida Limnohydrina gerechnet (Limnomedusae bei Kramp 1959, 1961). Wie aber REEs hervorgehoben hat, ist diese Einordnung nicht aufrechtzuerhalten; denn der Besitz eines Tricnidom aus Desmonemen (3), atrichen Haplonemen (4) und Stenotelen (19) bei Moerisia lyonsi spricht dafür, daß die Familie in die Hydroida Athecata eingereiht werden muß. Speziell der Besitz von Stenotelen ist ein eindeutig unterscheidendes Merkmal gegenüber allen Limnohydrina.

Auf Grund der Nesselzellausstattung gelang es ferner, der Unterordnung $\mathrm{Ha}$ lammohydrina die ihr zukommende Stellung im System zuzuweisen (WERNER 1965).

Ein Beispiel, das sich auf noch unveröffentlichte Befunde stützt, ist geeignet, die Bedeutung der speziellen Qualität der Kapseltypen besonders deutlich zu machen. Laut Tabelle 7 besitzen die Limnohydrina den Kapseltyp der makrobasischen Eurytelen (18). Er wurde bisher von Russell (1938) (vgl. Anmerkung 4, p. 8, Tab. 1) und Hand (1954a) für die Familie Proboscidactylidae signalisiert, noch nicht aber für die Familie 
Olindiidae. Nach eigenen Untersuchungen konnte inzwischen für 3 Species, nämlich Gonionemus vertens (syn. murbachi), Scolionema suvaense, sowie für eine noch nicht einwandfrei bestimmte Art der Gattung Gonionemus von Neapel der Besitz von makrobasischen Eurytelen (18) nachgewiesen werden. Die Polypen dieser Arten besitzen übereinstimmend ein Monocnidom von mikrobasischen Eurytelen (15), während die Medusen ein Tricnidom von atrichen Haplonemen (4), mikro- und makrobasischen Eurytelen $(15,18)$ aufweisen (vgl. Abb. 15 und 18). Es ist von großem Interesse, daß der spezielle Typ der makrobasischen Eurytelen (18) nur bei den frisch vom Polypen abgelösten Jungmedusen gefunden wird, und zwar nur auf ihrer Exumbrella, nicht aber bei erwachsenen Exemplaren. Dieser Typ ist an der Exumbrella der Jungmeduse funktionsfähig, wird aber verbraucht und nicht mehr ersetzt. Nach HADžI (1911) ist die Exumbrella der sich am Polypen entwickelnden Medusenknospe das Organsystem, in dem Nesselzellen zuerst gebildet werden. Der vorliegende Sachverhalt ist im Hinblick auf den Befund von HADŽI meines Erachtens nur in evolutionistischem Sinne zu deuten und damit zu erklären, daß die Olindiiden von Vorfahren abstammen, die den Typ der makrobasischen Eurytelen ebenfalls besessen haben und bei denen er zur Dauerausstattung gehört hat. Die Zusammenfassung der Olindiidae und Proboscidactylidae $\mathrm{zu}$ einer einheitlichen Gruppe findet damit in der Nesselzellausstattung eine Stütze.

Mit einem letzten Beispiel schließlich werden ebenfalls einige unveröffentlichte Beobachtungen yorweggenommen. WeILL (1934) und Russell $(1938,1939,1940$, vgl. auch 1953, p. 16) sind zu dem übereinstimmenden Ergebnis gekommen, daß bei den Hydroida Thecata (Calyptoblastes, Leptomedusae) die Nesselzellverhältnisse wenig geeignet erscheinen, zur Klärung der verwandtschaftlichen Beziehungen beizutragen, so daß die auch nach den morphologischen Merkmalen schwierige Gruppierung nicht die gewünschte Präzisierung erfahren konnte. Das liegt zweifellos in erster Linie an der geringen Zahl überhaupt vorhandener verschiedener Nesselkapseltypen. Durch die Entdeckung des einheitsspezifischen Typs der merotrichen Haplonemen (6) (Abb. 6) wurde aber wenigstens für einige Familien ein Bindeglied nachgewiesen. Dieser Typ wurde zuerst aufgefunden bei der Meduse von Eucheilota maculata (Fam. Lovenellidae), wo er auf die Endkolben der Cirren beschränkt ist, die sich an der Basis der Schirmrandtentakel befinden und deren Vorhandensein ein systematisches Merkmal darstellt. Außer in der Familie Lovenellidae kommen Cirren am Schirmrand auch in der Familie Eirenidae bei Helgicirrha und Pbialopsis vor, ferner in der Familie Eutimidae bei Eutima. Die Nesselzellausstattung von Arten der Gattungen Helgicirha und Phialopsis konnte noch nicht untersucht werden, wohl aber die von zwei Arten der Gattung Eutima, nämlich von Eutima gracilis und Eutima (syn. Octorchis) gegenbauri. Bei beiden Arten fand sich an den Endkolben der Cirren der gleiche Typ der merotrichen Haplonemen, so daß nach dem Merkmal ihres Vorhandenseins die Lovenellidae und Eutimidae zweifellos eine verwandtschafliche Beziehung erkennen lassen, die in der bisherigen Anordnung (vgl. Russell 1953, KRAMP 1961) noch nicht zum Ausdruck kommt. Für den Fall, daß sich dieser spezielle Kapseltyp, der offenbar nur in den Cirren vorhanden ist, auch bei den Eireniden nachweisen läßt, müßten die drei Familien Lovenellidae, Eirenidae, Eutimidae als miteinander verwandt angesehen werden. Mit den beiden letzten Beispielen bestätigt sich erneut, daß die spezielle Qualität der Kapseltypen zusätzliche positive Merkmale für ein natürliches System liefern kann. 


\section{DISKUSSION DER ERGEBNISSE}

In den Ubersichten der Tabellen 2 bis 7 ist das bislang vorliegende nachprüfbare Tatsachenmaterial enthalten, das die qualitative und quantitative Verteilung der Nesselkapseltypen auf die untersuchten systematischen Einheiten angibt. Es ist nicht ausgeschlossen, daß mit der Zunahme der speziellen Kenntnisse in Zukunft Ergänzungen oder Berichtigungen notwendig werden. Diese sind aber voraussichtlich nur gradueller, nicht prinzipieller Natur. Denn alle neueren Autoren haben die von WeILL geschaffene Tatsachenbasis bestätigt, so daß sie eine zuverlässige Grundlage für die Frage nach der Bedeutung der Nesselzellausstattung für die Systematik und Evolution der Cridaria und ihrer Untereinheiten darstellt. Der abschließenden und zusammenfassenden Diskussion der erzielten Ergebnisse, die grundsätzlich zu einer Bestätigung der Resultate Weilus geführt haben, sind die allgemeinen Regeln voranzustellen, die der Autor in sehr ausführlicher Form herausgearbeitet hat (1934, p. $636 \mathrm{ff}$.); sie beziehen sich auf den taxonomischen Wert 1. der Nesselkapsel als solcher und der verschiedenen Typen, 2. des Cnidom. Für unsere $Z$ wecke genügen die folgenden.

a) Form und Größe der Nesselkapseln sind ohne taxonomischen Wert. Diese Regel bezieht sich auf die Dimension und Umrißform der unentladenen Kapsel; der gleiche Typ kann in verschiedenen Größen- und Formvarietäten auftreten.

b) Kein Kapseltyp kommt bei a 11 e n Cnidariern der höheren oder niederen Einheiten vor.

c) Der taxonomische Wert der einzelnen NK-Typen ist verschieden, je nachdem sie auf einem systematischen Niveau bei allen oder nur bei einigen Einheiten einer bestimmten Gruppe vorkommen. Werct kommt so zur Unterscheidung von stabilen und instabilen Typen. Ein in einer Gruppe eines bestimmten Niveaus stabiler Typ kann auf einem anderen Niveau instabil sein.

d) Von taxonomischem Wert sind insbesondere die für eine Gruppe charakteristischen, also einheitsspezifischen Kapseltypen. Durch ihren Besitz schließen sich Einheiten zu natürlichen Gruppen zusammen.

e) Die Zahl der bei einer Species vorkommenden Kapseltypen ist beschränkt; sie wird von Werlu die Valenz des Cnidom genannt. Nach den bisherigen Ergebnissen lassen sich für eine Einzelart der Hydrozoa (die Siphonophore Forskalia, nach WEILL 1934, p. 510 f.) maximal 5 verschiedene Typen nachweisen, so daß die Hydrozoa höchstens ein Pentacnidom besitzen; die durchschnittliche Maximalzahl ist 4. Bei den Scyphozoa ist die Maximalzahl 3, bei den Anthozoa meist 4, bei Arten der Gattung Diadumene (Actiniaria) sogar 6 (CARLGREN 1940, p. 37 ff., 1945, p. 23). Wenn man die Spirocysten hinzurechnet, muß man dieser Gattung maximal ein Heptacnidom zuerkennen. Die Valenz eines Cnidom ist allgemein ohne taxonomischen Wert.

f) Das Cnidom einer Art ist konstant und genetisch fixiert; jede Art verfügt also nur über ein bestimmtes Muster verschiedener NK-Typen. Andere Typen treten bei ihr nicht auf. Wenn mehrere Typen bei einer Art vorhanden sind, so ist ihr relatives Mengenverhältnis ebenfalls konstant. Diese Regel schließt eine begrenzte Variabilität nicht aus, die individueller Natur ist oder vom Entwidklungszustand und äußeren Faktoren abhängt. So kann beispielsweise die Nesselzellausstattung von Polyp und Meduse der gleichen Art verschieden sein, wenn auch beide Generationen mindestens einen Typ 
gemeinsam haben. Ferner kann es bei Hungertieren zu einer zahlenmäßigen Verringerung bis zum völligen Verschwinden eines Kapseltyps kommen.

g) Keine systematische Einheit besitzt ein Muster von NK-Typen, die in ihrer Gesamtheit ausschließlich bei ihr vorkommen.

h) Das Cnidom von Arten, die sich im System fernstehen, kann identisch sein. Dafür ein Beispiel: Eudendrium ramosum (Hydrozoa Hydroida Athecata) und Lucernaria campanulata (Scyphozoa Stauromedusae) haben beide ein Cnidom aus atrichen Haplonemen (4) und mikrobasischen Eurytelen (15) (WeILL 1934, p. 388 f., 534 f.).

i) Die Nesselzellausstattung ist kein adaptives Merkmal, das heißt aus dem Cnidom einer Art kann nicht auf ihre Lebensweise oder ihr Biotop geschlossen werden und umgekehrt. Diese Regel gilt ohne Zeifel für das Speciesniveau. Wir werden aber später zu prüfen haben, ob auf höheren systematischen Niveaus der Nesselzellausstattung nicht doch auch ein allgemeiner adaptiver Charakter zugeschrieben werden muß (siehe p. 34).

Mit den vorstehenden Regeln wird zunächst das allgemeine Ergebnis detailliert, daß keine Einheit, von den Species bis zu den Klassen, qualitativ und quantitativ durch die Nesselzellausstattung eindeutig positiv gekennzeichnet werden kann. Weiterhin hat die Untersuchung der qualitativen und quantitativen Verteilung der Nesselkapseltypen auf den geprüften Niveaus der Klassen, Unterklassen, Ordnungen und Unterordnungen ergeben: 1. Die Zahl der in den systematischen Gruppen vorkommenden NK-Typen ist kein Merkmal von allgemeiner systematischer oder evolutionistischer Bedeutung. 2. Die allgemeine Differenzierungshöhe der in den systematischen Gruppen vorkommenden Kapseltypen ist kein Merkmal von systematischer oder evolutionistischer Bedeutung; für die Differenzierungshöhe werden als einfache Typen die Haplonemen, als höher differenzierte Typen die Heteronemen unterschieden. 3. Der Besitz von speziellen Kapseltypen ist von positivem systematischem und evolutionistischem Wert, das heißt, es ist in vielen (nicht in allen) Fällen möglich, aus dem Besitz spezieller Kapseltypen auf die Zugehörigkeit zu einer Einheit der höheren und niederen systematischen Niveaus zu schließen und von hier aus evolutionistische Entwicklungslinien aufzufinden.

Bekanntlich läßt sich der umfassendere oder begrenztere Wert eines systematischen Merkmals an seinem Voraussagewert für eine größere oder kleinere Gruppe von Species oder anderen systematischen Einheiten ablesen. Die mit dem Besitz der Nesselkapseln für die Systematik gegebene, nur beschränkte und relative Situation läßt sich auch so formulieren, daß als einzig sichere voraussagbare Eigenschaft nur diese zutrift: alle Cnidaria haben Nesselzellen, beziehungsweise jeder Cnidarier besitzt mindestens einen Typ von Nesselkapseln. Die spezielle Qualität des Nesselkapseltyps ist aber allgemein nicht voraussagbar, sondern nur in bestimmten Fällen und nur auf bestimmten Niveaus. So ist die positive Aussage: jedes Hydrozoon hat den oder jenen Nesselkapseltyp, nicht möglich; es kann nur negativ festgestellt werden, welche Typen bei einem Hydrozoon nicht vorkommen (vgl. Tab. 2). Entsprechendes gilt für die Ordnungen und Unterordnungen. Erst für die beiden Sectionen Capitata und Filifera (Athecata) ist eine positive Charakterisierung möglich: jede capitate Form hat Stenotelen (19), jede filifere Art hat mikrobasische Eurytelen (15) (vgl. Russell 1953, Rees 1957b). Bei den Scyphozoa mit ihrer insgesamt nur geringen Zahl von 3 Kapseltypen 
ist die Situation anders. Bei ihnen ist nach den bisherigen Kenntnissen die positive Aussage möglich: jedes Scyphozoon hat mikrobasische Eurytelen (15). Für die negative Charakterisierung wäre bei dieser Klasse laut Tabelle 2 eine große Zahl verschiedener Kapseltypen zu nennen, die ihr fehlen.

Für sämtliche Anthozoa ist in entsprechender Weise die positive Voraussage des Besitzes eines gemeinsamen Kapseltyps ebenfalls nicht möglich. Für die Unterklassen liegt die Situation verschieden: alle Octocorallia haben atriche Haplonemen (4), was eine Selbstverständlichkeit ist, da bei ihnen nur dieser eine Kapseltyp existiert. Ferner gilt: alle Hexacorallia haben Spirocysten (21); für die eigentlichen Kapseltypen aber ist keine positive, sondern nur eine negative Voraussage möglich. Eine positive Voraussage kann erst wieder für niedere Niveaus gemacht werden. Die größere oder geringere Voraussagemöglichkeit hinsichtlich des gemeinsamen Besitzes spezieller Kapseltypen bei allen Angehörigen einer Einheit ist daher umgekehrt proportional der Menge der verschiedenen Kapseltypen, über die sie auf dem betreffenden systematischen Niveau verfügt. Das kennzeichnet am besten die Lage, die sich für die Systematik aus der Nesselzellausstattung ergibt und die in den meisten Fällen die Prüfung der besonderen Situation notwendig macht. Grundsätzlich aber ließ sich zeigen, daß in vielen Fällen die Nesselzellausstattung durch die spezielle Qualität der Nesselkapseln zusätzliche systematische Merkmale liefern kann. Aus diesem Grunde ist es erforderlich, daß die Nesselzellausstattung möglichst aller bekannten Arten ermittelt wird und daß in jede Neubeschreibung auch die vorgefundenen Nesselkapseltypen aufgenommen werden.

Uber die bisherigen Aussagen hinaus erscheint eine weitere wesentliche Schlußfolgerung möglich zu sein, die meines Wissens bisher in ihrer Bedeutung nicht erkannt worden ist. Sie geht aus von der naheliegenden Frage, wie die erheblichen Unterschiede zu erklären sind, die bei den Einheiten der verschiedenen systematischen Niveaus in der Nesselzellausstattung, also in der qualitativen und quantitativen Verteilung der Kapseltypen bestehen. Die Frage nach den Ursachen der qualitativen Verteilung, warum bei den systematischen Einheiten gerade die vorhandenen und keine anderen Typen ausgebildet sind, warum also bestimmte Typen auf bestimmte Einheiten beschränkt sind, läßt sich, soviel ich sehe, zur Zeit nicht beantworten. Dagegen läßt sich eine plausible Erklärung für die Unterschiede in der quantitativen Verteilung finden, die in der Tabelle 8 besonders deutlich hervortreten; die Antwort besteht darin, daß auf den verschiedenen Niveaus ein positiver $Z$ us a m m e hang zwischen der quantitativen Verteilung der Kapseltypen bei den Einheiten und deren Formenmannigfaltigkeit besteht. Bei einer vergleichenden Gegenüberstellung der Klassen Hydrozoa, Scyphozoa und Anthozoa hinsichtlich ihrer Differenziertheit, das heißt hinsichtlich der Zahl morphologischer Baupläne, der Erscheinungen der Fortpflanzung und Entwicklung, der besiedelten Lebensräume kann kein Zweifel daran bestehen, daß die Hydrozoa weitaus an der Spitze stehen. Daß die Hydrozoa auch Brack- und Süßwässer besiedelt haben, während die Scyphozoa und Anthozoa fast vollständig auf das Meer beschränkt sind, ist ein Merkmal, das in diesem Zusammenhang besonders hervorzuheben ist. Von den neueren Autoren haben besonders HaDžr (1963) und UCHIDA (1963) in anderem Zusammenhang den ungleich größeren Reichtum der Hydrozoa an morphologischen Bauplänen und Lebenserscheinungen herausgestellt und eingehend diskutiert. Wenn 
auf der anderen Seite die Hydrozoa die größte Zahl verschiedener Nesselkapseltypen besitzen, wenn sie gleichzeitig auch über die größte Zahl klasseneigener Typen verfügen, Merkmale, von denen wir wissen, daß sie keinen allgemein gültigen Wert für die Systematik und Evolution haben, so ist das sicher kein Zufall, sondern muß offenbar eben mit ihrer größeren Mannigfaltigkeit an Formen und Lebenserscheinungen erklärt werden. Die Scyphozoa sind eine viel einheitlichere und weniger differenzierte Gruppe; damit stimmt überein, daß wir bei ihnen nur wenige verschiedene Kapseltypen und keinen einzigen klasseneigenen Typ finden. Daß die Anthozoa insgesamt eine formenreichere Gruppe darstellen als die Scyphozoa, dürfte ebenfalls außer Frage stehen, so daß sich ihre größere Zahl von NK-Typen und der Besitz von 2, die Spirocysten einbezogen, von 3 klasseneigenen Typen ebenfalls in den behaupteten Zusammenhang mit der größeren Differenziertheit einfügt.

Wenn diese Annahme Gültigkeit hat, so muß sie sich auf den anderen systematischen Niveaus verifizieren, es muß sich also die gleiche Korrelation nachweisen lassen. Die Hexacorallia und unter ihnen die Actiniaria haben ohne Frage den größeren Formenreichtum vor den Octocorallia beziehungsweise vor den anderen Ordnungen der Hexacorallia. Auf diese Vorrangstellung der Actiniaria hat CARLgren 1940 (p. 52 f.) aufmerksam gemacht; ihr entspricht, daß bei ihnen die meisten verschiedenen NK-Typen auftreten. Innerhalb der Hydrozoa sind die Hydroida als die formenreichste Gruppe anzusprechen, während die Trachylida mit den Unterordnungen der Trachy- und Narcomedusae eine in sich recht einheitliche Gruppe bilden, sowohl was ihre Baupläne als auch was ihren Lebensraum betrifft. Die Siphonophoren andererseits müssen als eine sehr differenzierte Gruppe gelten; darauf wird besonders von HADžI (1963, p. 109) mehrfach hingewiesen. In Ubereinstimmung damit haben sie ein reiches Muster von verschiedenen NK-Typen und besitzen auch einheitsspezifische Kapseltypen, während die Trachylida nur wenige NK-Typen und keinen einheitsspezifischen Typ aufweisen.

Schließlich läßt sich die gleiche positive Korrelation zwischen der Differenziertheit der Einheiten und der Zahl der Kapseltypen auch auf dem Niveau der Unterordnungen bei den Hydroida erkennen, bei denen den Athecata der Rang der differenziertesten Gruppe zuerkannt werden muß. Zwar sind die Thecata den Athecata in dem Merkmal der Verzweigung und Stöckchenbildung voraus und im Zusammenhang damit in der Mannigfaltigkeit der peridermalen Bildungen, doch läßt die allgemeine Morphologie, vor allem auch die Formenmannigfaltigkeit der Medusengeneration, keinen Zweifel daran aufkommen, daß die Athecata als die im ganzen reicher differenzierte Gruppe zu betrachten sind. Das hat übrigens HADžr (1963, p. $237 \mathrm{ff}$.) dazu veranlaßt, die Thecata als die „more primitive“ Gruppe zu betrachten, eine Auffassung, die aber keineswegs zwingend erscheint. Hinsichtlich der Biotope sei noch daran erinnert, daß die Thecata auf Hartsubstrate angewiesen sind, während die Athecata auch Weichböden besiedeln und in der Familie Margelopsidae sogar mit der Polypengeneration das Pelagial erobert haben. So läßt sich auch bei den Athecata der größere Reichtum an Nesselkapseltypen mit ihrer größeren Mannigfaltigkeit an Bauplänen und Lebenserscheinungen erklären.

Die Ubersicht der Tabelle 8 spiegelt die positive Korrelation zwischen der Differenziertheit der Gruppen und der Zahl überhaupt verschiedener und überdies einheitsspezifischer Kapseltypen deutlich wider. Die gleiche Korrelation gilt auch für den 
qualitativen Aspekt der Differenzierungshöhe. Ein großer Anteil an den höher differenzierten Typen, also an Heteronemen wird bei den gleichen Gruppen gefunden, die ein Maximum an verschiedenen NK-Typen besitzen (siehe pp. $18 \mathrm{ff} ., 24 \mathrm{ff}$. und Tab. 8, 9).

Im Endergebnis gelangen wir damit zu dem Resultat, daß die qualitative und quantitative Verteilung der NK-Typen eine Funktion der Formenmannigfaltigkeit der systematischen Einheiten ist. In diesem Sinne muß die Nesselzellausstattung als adaptives Merkmal verstanden werden. Der Oberblick über die Tabelle 8 läßt erkennen, daß es auf den systematischen Niveaus jeweils die niedersten Einheiten sind, die über die größte Zahl verschiedener Nesselkapseltypen verfügen und die gleichzeitig die in morphologischer und biologischer Hinsicht differenziertesten Gruppen darstellen. Die letzte Ursache dafür dürfte darin zu suchen sein, daß die im System niederen Einheiten die größere Plastizität und Anpassungsfähigkeit besitzen. Daß diese Regel nicht uneingeschränkt gilt, zeigen die Siphonophoren, die als eine abgeleitete und gleichwohl als eine hoch differenzierte Gruppe zu betrachten sind.

In jedem Fall stehen die hier erzielten Resultate in Einklang mit dem bisher gültigen System und damit in Gegensatz zu der Auffassung von HADžI $(1958,1963)$, daß sich die Nesselzellausstattung der Cnidaria progressiv in Richtung AnthozoaHydrozoa entwickelt habe. Diese Annahme dient HADŽr als Stütze für die Auffassung, daß die bisher gültige Reihenfolge Hydrozoa-Scyphozoa-Anthozoa umgekehrt werden müsse, weil die Anthozoa als primitivste Gruppe von den Turbellaria abzuleiten sei. Bekanntlich hat die Hypothese von HADZ̆I, die er zuletzt 1963 in ausführlicher Form dargestellt hat, eine internationale Diskussion in Gang gebracht und ebenso Zustimmung wie Ablehnung gefunden (vgl. REMANE 1958, StEINBöck 1958a, b, HAND 1959, Ax 1961, Reisinger 1961).

$\mathrm{Zu}$ der Erörterung und Bewertung der morphologischen und entwicklungsgeschichtlichen Tatsachen kann hier nicht Stellung genommen werden; vielmehr muß ich mich auf die Nesselzellverhältnisse und ihre Beurteilung durch HaDžr beschränken. Der Autor gibt in detaillierter Form (1963, p. 201 ff.) die Gesichtspunkte an, die seiner Meinung nach zu der Konsequenz führen, daß die Nesselzellausstattung der Anthozoa als die ursprünglichste, die der Hydrozoa aber als höchst differenzierte zu betrachten sei; insbesondere erörterte er die Merkmale, die nach seiner Auffassung progressiver beziehungsweise regressiver Natur seien. Ohne hier in nähere Einzelheiten einzugehen, ist dem Autor folgendes entgegenzuhalten.

a) Die tatsächliche qualitative und quantitative Verteilung der NK-Typen ist nicht mit der von HADžx behaupteten systematischen Reihenfolge in Einklang zu bringen. Müßte man nicht die Scyphozoa nach ihrer Nesselzellausstattung als die ursprünglichere Klasse bezeichnen, und müßte man nicht bei den Hydrozoa aus dem gleichen Grunde die Trachylida, die sicherlich eine abgeleitete Gruppe darstellen, an die Basis stellen? Es erscheint nicht zulässig, bei den Gruppen mit reicher Nesselzellausstattung mit progressiver Entwicklung, bei denen mit zahlenmäßig geringer Nesselzellausstattung mit regressiver Entwicklung zu operieren. Das hat HADŽr (1963, p. 203) speziell bei den Trachyliden getan, denen er ein Monocnidom zuschreibt. Wir wissen aber heute, daß die Trachyliden über drei Nesselkapselarten verfügen, unter denen sich der hochspezialisierte Typ der Stenotelen (19) befindet.

b) In der vorliegenden Untersuchung ist der eindeutige Beweis dafür erbracht 
worden, daß die quantitative Verteilung und die Differenzierungshöhe der NK-Typen keine Merkmale von allgemein gültigem systematischem und evolutionistischem Wert sind, sondern durch die positive Korrelation mit der Formenmannigfaltigkeit der systematischen Einheiten ihre natürliche und den Tatsachen entsprechende Erklärung finden. Daß die Nesselzellausstattung in diesem Sinn als adaptives Merkmal betrachtet werden muß, das sehr wahrscheinlich in kausalem Zusammenhang mit der größeren Anpassungsfähigkeit und Plastizität der Gruppen steht, die über die reichste Nesselzellausstattung verfügen, entzieht der Hypothese von HADŽI die Grundlage, der in den Nesselzellverhältnissen ein Merkmal von allgemeiner evolutionistischer Bedeutung sehen will. Die den Tatsachen entsprechende Bewertung der Nesselzellverhältnisse gibt keine Veranlassung, sie als Stütze für eine Umkehrung des bisher gültigen Systems anzuführen.

Zum Schluß ist darauf hinzuweisen, daß für die Nesselzellverhältnisse der in dieser Arbeit noch nicht berücksichtigten Niveaus der Familien, Gattungen und Arten nach den vorliegenden speziellen Untersuchungen keine prinzipiell anderen Resultate zu erwarten sind als für die höheren systematischen Einheiten. Insbesondere gilt auf dem Species-Niveau, daß manche Arten einer Gattung sich durch ihr Cnidom unterscheiden, wobei oftmals auch zusätzliche feinere Unterschiede, wie Form und Größe der Nesselkapseln, berücksichtigt werden müssen (vgl. PAPENfuss 1936). In anderen Fällen aber lassen sich keine Unterschiede ermitteln oder sie sind so gering, daß Species und Gattungen nicht nach ihren Nesselzellverhältnissen getrennt werden können. Überdies spielt auch die persönliche Erfahrung und Übung bei allen Untersuchungen über die Nesselkapseln eine wesentliche Rolle. Bei Anwendung geeigneter Methoden, wobei besonders die Untersuchung von Quetschpräparaten lebendfrischen Materials mit Hilfe des Phasenkontrastverfahrens zu nennen ist, lassen sich nicht nur die Kapseltypen bekannter Species unterscheiden, sondern es gelingt auch oft, eine unbekannte Art in die zugehörige niedere und höhere systematische Einheit einzuordnen. Nach den eigenen Erfahrungen sind etwa die Nesselkapseln der thecaten Hydroiden sowohl nach den Typen wie auch nach den Größen- und Formverhältnissen so charakteristisch, daß die Unterscheidung von den Athecata in den meisten Fällen ohne Schwierigkeit möglich ist. Für den Systematiker ist somit die Berücksichtigung der Nesselzellen ein wesentliches und unerläßliches Hilfsmittel. Anzustreben ist für die Zukunft, einen Katalog der Nesselzellausstattung der Species zusammenzustellen, für die sie bekannt ist. In der von den meisten Autoren bereits geübten Weise sollten dabei außer den Typdiagnosen stets auch Größenangaben der Nesselkapseln (Längs- und Querdurchmesser) aufgeführt werden.

\section{ZUSAMMENFASSUNG}

1. Die Klassifikation der verschiedenen Kategorien von Nesselkapseln nach WEILL (1934) wird beschrieben. Sie beruht auf morphologischen Merkmalen der entladenen Nesselkapseln und umfaßt 20 verschiedene Typen, von denen 2 neu eingefuhrt werden.

2. Nach den speziellen Befunden der früheren Autoren, besonders denen von WeILL, 
Russell, Carlgren, Itô \& Inoue und nach eigenen Beobachtungen wird die qualitative und quantitative Vertellung der Nesselkapseltypen auf die systematischen Einheiten untersucht. Berücksichtigt werden die Niveaus der Klassen, Unterklassen, Ordnungen, für die Ordnung Hydroida auch die Unterordnungen.

3. Die Prüfung der Nesselzellverhältnisse auf ihre allgemeine Bedeutung für Systematik und Evolution ergibt folgende Resultate, mit denen die detaillierten Erörterungen von WEILL bestätigt und ergänzt werden:

a) Die qualitative und quantitative Verteilung der Kapseltypen auf die systematischen Einheiten enthält keine Merkmale von absolut gültigem Wert.

b) Die Differenzierungshöhe der in den verschiedenen Einheiten gefundenen Kapseltypen, die nach dem quantitativen Anteil von einfachen (Haplonemen) und höher differenzierten Typen (Heteronemen) bewertet wird, ist kein Merkmal von systematischer und evolutiver Bedeutung.

c) Der Besitz von speziellen Kapseltypen ist ein Merkmal von positiver, aber je nach dem geprüften Niveau begrenzter und relativer Bedeutung. Mit dem Besitz der speziellen Kapseltypen sind damit systematische Merkmale gegeben, die neben den morphologischen und entwicklungsgeschichtlichen Merkmalen für ein natürliches System Verwendung finden können. Nach den bisherigen Kenntnissen stehen die Nesselzellverhältnisse mit dem bestehenden System in Übereinstimmung und stützen es. In unsicheren Fällen, in denen die Eingruppierung nach morphologischen Merkmalen allein zweifelhaft bleibt, kann die spezielle Qualität der Nesselkapseln die Entscheidung über die systematische Stellung ermöglichen. Dafür werden aus der Literatur und nach eigenen Beobachtungen instruktive Beispiele gegeben.

4. Zwischen der qualitativen und quantitativen Reichhaltigkeit der Nesselzellausstattung einer systematischen Gruppe und ihrer allgemeinen Differenziertheit - also ihrer Mannigfaltigkeit an Bauplänen, Lebens- und Entwicklungserscheinungen besteht eine positive Korrelation. Eine in dieser Hinsicht differenzierte Gruppe hat eine reichere Nesselzellausstattung als eine weniger differenzierte. Aus dieser Korrelation erklärt sich, daß die Hydrozoa als die in morphologischer, entwicklungsgeschichtlicher und ethologischer Hinsicht differenzierteste Klasse die größte Anzahl verschiedener Nesselkapseltypen haben.

5. Die Nesselzellverhältnisse bieten daher keine Stütze für eine Umkehrung des bisher gültigen Systems.

Herrn Professor Dr. R. WerLI, Bordeaux, danke ich herzlich für das liebenswürdige Einverständnis, eine Anzahl seiner Abbildungen und ein Originalphoto in diese Arbeit aufnehmen zu dürfen. Außerdem habe ich ihm für die Hilfe bei der Literaturbeschaffung und für die Möglichkeit der brieflichen Diskussion wesentlicher Fragen zu danken. Mein Dank gebührt weiterhin Herrn Dr. G. B. Chapman, New York, der mir ebenfalls ein Originalphoto zur Verfügung gestellt hat, sowie Herrn Dr. W. Vervoort, Leiden, für die Einsichtnahme in sein im Druck befindliches Manuskript. Schließlich möchte ich meiner langjährigen Mitarbeiterin Frau H. JaEger-Schmot Dank und Anerkennung für die unermüdliche Mithilfe aussprechen. 


\section{ZITIERTE LITERATUR}

Ax, P., 1961. Verwandtschaftsbeziehungen und Phylogenie der Turbellarien. Ergebn. Biol. 24, $1-68$.

Borsseau, J. P., 1952. Recherches sur l'histochimie des Cnidaires et de leurs nématocystes. Bull. Soc. zool. Fr. 87, 151-169.

Boulllon, J., 1957. Etude monographique du genre Limnocnida (Limnomedusae). Annls. Soc. r. zool. Belg. 87, 253-500.

BrincRMANN, A., 1964. Observations on the structure and development of the medusa of Velella velella (Linné 1758). Vidensk. Meddr dansk naturh. Foren. 126, 327-336.

Cartgren, O., 1940. A contribution to the knowledge of the structure and distribution of the cnidae in the Anthozoa. Lunds Univ. Arsskr., N. F. (Avd. 2) 36, 1-62.

- 1945. Further contributions to the knowledge of the cnidom in the Anthozoa, especially in the Actiniaria. Lunds Univ. Arsskr., N. F. (Avd. 2) 41, 1-24.

Chapman, G. B., 1961. The fine structure of the stenoteles of Hydra. In: The biology of Hydra and of some other coelenterates. Ed. by H. M. Lenhoff \& W. F. Loomis. Univ. Miami pr., Coral Gables, Fla, 131-151.

- \& Tilney, L. G., 1959 a. Cytological studies of the nematocysts of Hydra. 1. Desmonemes, Isorhizas, cnidocils, and supporting structures. J. biophys, biocbem. Cytol. 5, 69-78.

- 1959 b. Cytological studies of the nematocysts of Hydra. 3. Stenoteles. J. biophys, biochem. Cytol. 5, 79-84.

Cutress, CH. E., 1955. An interpretation of the structure and distribution of cnidae in Anthozoa. Syst. Zool. 4, 120-137.

EwER, R. F., 1947. On the functions and mode of action of the nematocysts of Hydra. Proc. zool. Soc. Lond. 117, 365-376.

Hadžı, J., 1911 a. Uber die Nesselzellverhältnisse bei den Hydromedusen. Zool. Anz. 37, $471-478$.

- 1911 b. Razmjestaj i selidba knidocita u hidromeduza $\mathrm{i}$ u hidroida nopce. Rad jugosl. Akad. Znan. Umjetn. 188, 139-247.

- 1958. Zur Diskussion über die Abstammung der Eumetazoen. Zool. Anz. (Suppl. Bd) 21, $169-179$.

- 1963. The Evolution of the Metazoa. Pergamon pr., Oxford, $499 \mathrm{pp}$.

HAND, C., 1954 a. Three pacific species of "Lar" (including a new species), their hosts, medusae, and relationships. (Coelenterata, Hydrozoa.) Pacif. Sci. 8, 51-67.

- $1954 \mathrm{~b}$. The sea anemones of Central California. Part. I. The Corallimorpharian and Athenarian anemones. Wasmann J. Biol. 12, 345-375.

- 1955 a. The sea anemones of Central California. 2. The Endomyarian and Mesomyarian anemones. Wasmann J. Biol. 13, 37-99.

- 1955 b. The sea anemones of Central California. 3. The Acontarian anemones. Wasmann J. Biol. 13, 189-251.

- 1959. On the origin and phylogeny of the Coelenterates. Syst. Zool. 8, 191-202.

- 1961. Present state of nematocyst research: Types, structure, and function. In: The biology of Hydra and of some other coelenterates. Ed. by H. M. Lenhoff \& W. F. Loomis. Univ. Miami pr. Coral Gables, Fla, 187-202.

- \& Hendrickson, J. R., 1950. A two-tentacled, commensal hydroid from California (Limnomedusae, Proboscidactyla). Biol. Bull. mar. biol. Lab., Woods Hole 99, 74-87.

Huvé, P., 1954. Hydranthea et Campalecium, genres méditerranéens aberrants d' hydroides de la famille des Haleciides. Recl. Trav. Stn. mar. Endoume 13, 173-192.

ITô, T. \& Inoue, K., 1962. Systematik studies on the nematocysts of Cnidaria I. Nematocysts of Gymnoblastea and Calyptoblastea. Mem. Ehime Univ. (Science B) 4, 445-460.

Kaestner, A., 1965. Lehrbuch der speziellen Zoologie. Bd. I. Wirbellose. 1. T. G. Fischer, Jena, $845 \mathrm{pp}$.

Kramp, P. L., 1938. Die Meduse von Ostroumovia inkermanika (Pal.-Ostr.) und die systematische Stellung der Olindiiden. Zool. Anz. 122, 103-108. 
- 1959. The Hydromedusae of the Atlantic Ocean and adjacent waters. Dana Rep. 46, 1-283.

- 1961. Synopsis of the medusae of the world. J. mar. biol. Ass. U. K. 40, 1-469.

Küнn, A., 1914, 1915, 1916. Coelenterata (Hohltiere). Bronn's Kl. Ordn Tierreichs 2 (Abt. 2), 403-538.

Lenhoff, H. M. \& Looms, W. F., 1961. The biology of Hydra and of some other coelenterates. Univ. Miami pr., Coral Gables, Fla, 467 pp.

MERGNER, H., 1964. Unsere Kenntnise über Bau und Funktion der Nesselzellen bei Hydroiden. Natur-Mus., Frankfurt 94, 15-32.

Naumov, D. V., 1960. Hydroiden und Hydromedusen der Meeres-, Brackwasser- und Süßwasserbecken der USSR. (Russ.) Opredeliteli faune SSSR. Zool. Muz. Akad. nauk. 70, $1-585$.

Papenfuss, E. J., 1936. The utility of nematocysts in the classification of certain Scyphomedusae. 1. Lunds Univ. Arsskr., N. F. (Avd. 2) 31, 1-26.

Pax, F., 1935. Anthozoa. In: Grimpe, G. \& E. Wagler (Begr.): Tierwelt der Nord- u. Ostsee. Akad. Verl. Ges., Leipzig, Bd. (T.) 1 (3 e 2), 81-317.

- 1940. Anthozoa. Bronn's Kl. Ordn. Tierreichs 2 (Abt. 2), 177-336.

PICARD, J., 1955 a. Nouvelles recherches sur les hydroméduses des herbiers méditerranéens de Posidonies. Recl. Trav. Stn. mar. Endonme 15, 59-71.

- 1955 b. Les hydroides Pteronematidae, origine des "Siphonophores" Chondrophoridae. Bull. Inst. océanogr. Monaco 52 (1059), 1-8.

- 1957. Etudes sur les hydroides de la superfamille Pteronematoidea. Bull. Inst. océanogr. Monaco 54 (1106), 1-12.

Proken, L. E. R., 1957. Stinging capsules and designing nature. New Biol. 22, 56-72.

ReEs, W. J., 1957 a. Status of Moerisia lyonsi Boulenger and related hydroids. Nature, Lond. 180,445 .

- 1957 b. Evolutionary trends in the classification of capitate hydroids and medusae. Bull. Br. Mus. nat. Hist. (Zool.) 4, 455-534.

Remane, A., 1958. Zur Verwandtschaft und Ableitung der niederen Metazoen. Zool. Anz. (Suppl. Bd) 21, 179-196.

Reisinger, E., 1961. Morphologie der Coelenteraten, acoelomaten und pseudocoelomaten Würmer. Fortschr. Zool. 13, 1-82.

Russell, F.S., 1938. On the nematocysts of Hydromedusae. J.mar. biol. Ass. U. K. 23, 145-165.

- 1939. On the nematocysts of Hydromedusae. 2. J. mar. biol. Ass. U. K. 23, 347-359.

- 1940. On the nematocysts of Hydromedusae. 3. J. mar. biol. Ass. U. K. 24, 515-523.

- 1953. The medusae of the British isles. Univ. pr., Cambridge, 530 pp.

Schulze, P., 1914. Bestimmungstabellen der deutschen Hydraarten. Sber. Ges. naturf. Fretunde Berl. 9, 395-398.

- 1922. Der Bau und die Entladung der Penetranten von Hydra attenuata Pallas. Arch. Zellforsch. 16, 383-438.

Stechow, E., 1923. Zur Kenntnis der Hydroidenfauna des Mittelmeeres, Amerikas und anderer Gebiete. Zool. Jb. (Syst.) 47, 29-270.

Sterniöck, O., 1958 a. Zur Phylogenie der Gastrotrichen. Zool. Anz. (Suppl. Bd) 21, 128-169.

- 1958 b. Schlußwort zur Diskussion Remane/Steinböck. Zool. Anz. (Suppl, Bd) 21, 196-218.

Stephenson, T. A., 1929. On the nematocysts of sea anemones. J. mar. biol. Ass. U. K. 16, $173-200$.

Thier, HJ., 1962. Clavopsella quadrularia nov. spec. (Clavopsellidae nov. fam.), ein neuer Hydroidpolyp aus der Ostsee und seine phylogenetische Bedeutung. Z. Morph. Ökol. Tiere $51,227-260$.

Threl, M. E., 1962. Bestimmungstabellen der Polypen und Medusen der russischen Gewässer. Mitt. hamburg. zool. Mus. Inst. 60, 285-324 (nach Naumov, D. V., 1960).

- 1964. Allgemeine Betrachtungen über die organismische Integration der Hydroiden, ihre phylogenetische Entwicklung, ihre Klassifikation und geographische Verbreitung in der UdSSR. Mitt. hamburg. zool. Mus. Inst. 61, 1-91 (nach NAUMov, D. V., 1960).

Trschierex, H., 1936. Die Nesselkapseln der Anthipatharien und ihre differentialdiagnostische Bedeutung. Diss. Breslau, $60 \mathrm{pp}$. 
Totron, A. K. \& Mackie, G. O., 1960. Studies on Physalia physalis (L.). 'Discovery' Rep. 30, 301-408.

UCHIDA, T., 1963. The systematic position of the Hydrozoa. Jap. J. Zool. 14, 1-14.

VERVOORT, W., 1965. Skeletal structure in the Solanderiidae and its bearing on hydroid classification. Im Druck.

WeILr, R., 1934. Contribution a l'étude des Cnidaires et de leurs nématocystes. 1. Recherches sur les nématocystes (morphologie - physiologie - développement). 2. Valeur taxonomique du cnidome. Trav. Stn. zool. Wimereux 10/11, 1-701.

- 1935. Halammobydra Remane, genre d'Hydrozoaires aberrant. Son cnidome et sa position taxonomique: affinité avec les Siphonophores Chondrophorides et avec Protohydra leuckarti Greeff. Bull. Soc. zool. Fr. 60, 73-87.

- 1964. Une nouvelle catégorie de nématocystes: existence, chez Apolemia uvaria Eschh., de nématocystes birhopaloides. C. r. hebd. Séanc. Acad. Si., Paris, 258, 4343-4344.

Werner, B., 1961. Morphologie und Lebensgeschichte, sowie Temperaturabhängigkeit der Verbreitung und des jahreszeitlichen Auftretens von Bongainvillia superciliaris (L. Agassiz). Helgoländer wiss. Meeresunters. 7, 206-237.

- 1965. Halammobydra Remane, Medusennatur und Stellung im System. Zool. Anz. (Suppl. Bd) 28, 163-178. 